Document downloaded from:

http://hdl.handle.net/10251/121414

This paper must be cited as:

Lucas Alba, S.; Gutiérrez Gil, R. (2018). Use of logical models for proving infeasibility in term rewriting. Information Processing Letters. 136:90-95.

https://doi.org/10.1016/j.ipl.2018.04.002

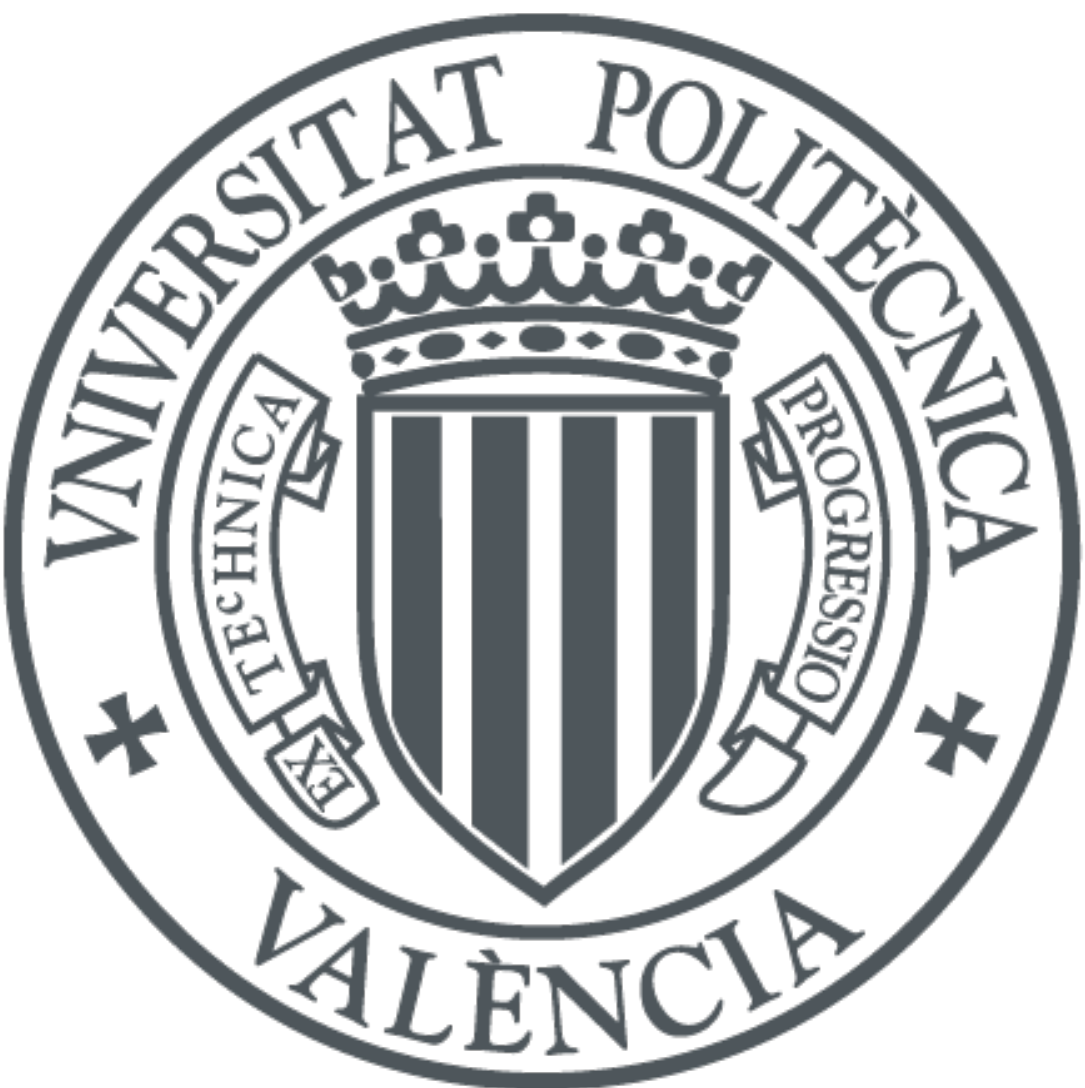

The final publication is available at

http://doi.org/10.1016/j.ipl.2018.04.002

Copyright Elsevier

Additional Information 


\title{
Use of Logical Models for Proving Infeasibility in Term Rewriting ${ }^{\text {th }}$
}

\author{
Salvador Lucas ${ }^{\mathrm{a}}$, Raúl Gutiérrez ${ }^{\mathrm{a}}$ \\ ${ }^{a}$ DSIC, Universitat Politècnica de València
}

\begin{abstract}
Given a (Conditional) Rewrite System $\mathcal{R}$ and terms $s$ and $t$, we consider the following problem: is there a substitution $\sigma$ instantiating the variables in $s$ and $t$ such that the reachability test $\sigma(s) \rightarrow_{\mathcal{R}}^{*} \sigma(t)$ succeeds? If such a substitution does not exist, we say that the problem is infeasible; otherwise, we call it feasible. Similarly, we can consider reducibility, involving a single rewriting step. In term rewriting, a number of important problems involve such infeasibility tests (e.g., confluence and termination analysis). We show how to recast infeasibility tests into the problem of finding a model of a set of (first-order) sentences representing the operational semantics of $\mathcal{R}$ together with some additional sentences representing the considered property which is formulated as an infeasibility test.
\end{abstract}

Keywords: Conditional rewriting, confluence, dependency graph, operational termination.

\section{Introduction}

Conditional Term Rewriting Systems (CTRSs) [19, Section 7] consist of rules $\ell \rightarrow r \Leftarrow c$, where the conditional part $c$ is a (possibly empty) sequence $s_{1} \approx$ $t_{n}, \ldots, s_{n} \approx t_{n}$ of conditions whose satisfaction is required before being allowed to apply a rewriting step with $\ell$ and $r$ in the usual way. Several interpretations of the satisfiability of conditions are possible [19, Definition 7.1.3]. For instance, dealing with oriented CTRSs, the evaluation of $c$ with respect to a substitution $\sigma$ consists of testing the instances of $s_{i}$ and $t_{i}$ for reachability, i.e., checking whether $\sigma\left(s_{i}\right)$ rewrites into $\sigma\left(t_{i}\right)$, written $\sigma\left(s_{i}\right) \rightarrow_{\mathcal{R}}^{*} \sigma\left(t_{i}\right)$, for all $1 \leq i \leq n$.

Given $n>0$, a sequence $\left(s_{i} \bowtie_{i} t_{i}\right)_{i=1}^{n}$ of rewriting goals $s_{i} \bowtie_{i} t_{i}$, where $s_{i}$ and $t_{i}$ are terms, and $\bowtie_{i}$ are predicate symbols $\rightarrow$ or $\rightarrow^{*}$ is called a feasibility sequence. Such a sequence is $\mathcal{R}$-feasible if there is a substitution $\sigma$ such that the instantiated goals $\sigma\left(s_{i}\right) \bowtie_{i} \sigma\left(t_{i}\right)$ are satisfied when $\bowtie_{i}$ is interpreted as the one-step or rewriting relations $\rightarrow_{\mathcal{R}}$ and $\rightarrow_{\mathcal{R}}^{*}$ for $\mathcal{R}$, respectively; otherwise, the sequence is called $\mathcal{R}$-infeasible (see Section 2 for a formal definition).

\footnotetext{
Partially supported by the EU (FEDER), Spanish MINECO project TIN2015-69175-C41-R and GV project PROMETEOII/2015/013.
} 
Example 1. Consider the CTRS $\mathcal{R}$ [19, Example 7.2.45]:

$$
\begin{aligned}
& \mathrm{a} \rightarrow \mathrm{a} \Leftarrow \mathrm{b} \approx x, \mathrm{c} \approx x \\
& \mathrm{~b} \rightarrow \mathrm{d} \Leftarrow \mathrm{d} \approx x, \mathrm{e} \approx x
\end{aligned}
$$

$$
\mathrm{c} \rightarrow \mathrm{d} \Leftarrow \mathrm{d} \approx x, \mathrm{e} \approx x
$$

where $\mathrm{a}, \ldots, \mathrm{e}$ are constants and $x$ is a variable. Since $\mathrm{d}$ and e are irreducible, the only way for $\mathrm{d} \rightarrow^{*} x, \mathrm{e} \rightarrow^{*} x$ to be $\mathcal{R}$-feasible is instantiating $x$ to both $\mathrm{d}$ and e at the same time, which is not possible. Thus, (2) and (3) cannot be used in any rewriting step. They are called infeasible rules and may be removed (without changing the induced rewrite relation). Actually, (1) is infeasible too.

$\mathcal{R}$-infeasibility can be used to (i) disable the use of conditional rules in reductions or even remove them, ${ }^{1}$ (ii) discard conditional dependency pairs $u \rightarrow v \Leftarrow c$ in the analysis of operational termination of CTRSs [12], (iii) discard conditional critical pairs $u \downarrow v \Leftarrow c$ that arise in the analysis of confluence of CTRSs $[19,20,21]$, (iv) prove root-stability of a term $t$ (i.e., the absence of any rewriting sequence from $t$ to an instance of a left-hand side $\ell$ of a rewrite rule $\ell \rightarrow r \Leftarrow c$ ) as the $\mathcal{R}$-infeasibility of $t \rightarrow^{*} \ell$ for each $\ell \rightarrow r \Leftarrow c \in \mathcal{R}$, (v) prove irreducibility of ground terms $t$ (which is undecidable for CTRSs) as the $\mathcal{R}$-infeasibility of $t \rightarrow x$ for a variable $x$, (vi) prove the non-joinability of terms $s$ and $t$ as the $\mathcal{R}$-infeasibility of $s \rightarrow^{*} x, t \rightarrow^{*} x$ (with $x$ not occurring in $s$ or $t$ ), or (vii) discard arcs in the dependency graphs that are obtained during the analysis of termination using dependency pairs, see, e.g., [2] for TRSs and [11] for CTRSs.

In Section 3, we prove that $\mathcal{R}$-infeasibility problems can be translated into the problem of finding a model $\mathcal{A}$ of the set of sentences $\overline{\mathcal{R}}$ representing the operational semantics of the CTRS $\mathcal{R}$ plus a sentence $\neg(\exists \vec{x}) \bigwedge_{i=1}^{n} s_{i} \bowtie_{i} t_{i}$ where all symbols (including $\rightarrow$ and $\rightarrow^{*}$ as predicate symbols) can be freely interpreted in a first-order structure $\mathcal{A}$. In Section 4 we show by means of examples how to apply our method to problems (i)-(vii). We assume familiarity with the basic notions, terminology and notations of (conditional) term rewriting (see, e.g., $[3,19]$ for TRSs and [19, Section 7] for CTRSs) and first-order logic [16].

The research in this paper was first presented in [8] (a restricted, nonsystematic use in proofs of operational termination of CTRSs is sketched in [7, Section 11.1]) and then settled by the first author in a first-order logic framework in [6]. In this paper we have extended the treatment of [8] to more general properties of rewrite systems (e.g., reducibility or root-stability, see Section 4.3). Also, in contrast to [6], we show that focusing on CTRSs and term rewriting enables the use of specific refinements available for CTRSs only (e.g., usable rules, see Section 2). This allows us to deal with more applications and examples. Interestingly, our semantic approach together with the aforementioned improvements applies to all the examples solved in papers developing different specific techniques to deal with problems (i)-(vii) $[1,17,20,21]$.

\footnotetext{
${ }^{1}$ Sometimes, infeasible rules cannot be removed without changing relevant properties of a CTRS. For instance, $\mathcal{R}=\{\mathrm{b} \rightarrow \mathrm{c}, \mathrm{a} \rightarrow \mathrm{b} \Leftarrow \mathrm{a} \approx \mathrm{b}\}$ is not operationally terminating (see Section 4.1 below) due to the conditional rule, which is infeasible. However, after removing it, an operationally terminating CTRS is obtained.
} 


$$
\frac{x \rightarrow y \quad y \rightarrow^{*} z}{x \rightarrow^{*} z}
$$

$$
\begin{gathered}
x_{i} \rightarrow y_{i} \\
\hline f\left(x_{1}, \ldots, x_{i}, \ldots, x_{k}\right) \rightarrow f\left(x_{1}, \ldots, y_{i}, \ldots, x_{k}\right) \\
\text { for all } f \in \mathcal{F} \text { and } 1 \leq i \leq k=\operatorname{arity}(f) \\
\frac{s_{1} \rightarrow^{*} t_{1} \cdots s_{n} \rightarrow^{*} t_{n}}{\ell \rightarrow r} \\
\text { for } \ell \rightarrow r \Leftarrow s_{1} \approx t_{1}, \ldots, s_{n} \approx t_{n} \in \mathcal{R}
\end{gathered}
$$

Figure 1: Inference rules for conditional rewriting with a CTRS $\mathcal{R}$ with signature $\mathcal{F}$

\section{Infeasibility problems}

Borrowing [19, Definition 7.1.8(3)] we introduce the following.

Definition 2. Let $\mathcal{R}$ be a CTRS. A sequence $\left(s_{i} \bowtie_{i} t_{i}\right)_{i=1}^{n}$, where $s_{i}$ and $t_{i}$ are terms and $\bowtie_{i} \in\left\{\rightarrow, \rightarrow^{*}\right\}$ for all $1 \leq i \leq n$ is called a feasibility sequence. It is $\mathcal{R}$-feasible if there is a substitution $\sigma$ such that, for all $1 \leq i \leq n, \sigma\left(s_{i}\right) \rightarrow_{\mathcal{R}} \sigma\left(t_{i}\right)$ if $\bowtie_{i}$ is $\rightarrow$, and $\sigma\left(s_{i}\right) \rightarrow_{\mathcal{R}}^{*} \sigma\left(t_{i}\right)$ if $\bowtie_{i}$ is $\rightarrow^{*}$. Otherwise, it is $\mathcal{R}$-infeasible.

In Definition 2 we write $s \rightarrow_{\mathcal{R}}^{*} t$ or $s \rightarrow_{\mathcal{R}} t$ for terms $s$ and $t$ iff there is a proof tree for $s \rightarrow^{*} t$ (resp. $s \rightarrow t$ ) using $\mathcal{R}$ in the inference system of Figure 1 [9]. All rules in the inference system in Figure 1 are schematic in that each inference rule $\frac{B_{1} \cdots B_{n}}{A}$ can be used under any instance $\frac{\sigma\left(B_{1}\right) \cdots \sigma\left(B_{n}\right)}{\sigma(A)}$ of the rule by a substitution $\sigma$. For instance, (Rl) actually establishes that, for every rule $\ell \rightarrow r \Leftarrow s_{1} \approx t_{1}, \ldots, s_{n} \approx t_{n}$ in the CTRS $\mathcal{R}$, every instance $\sigma(\ell)$ by a substitution $\sigma$ rewrites into $\sigma(r)$ provided that, for each $s_{i} \approx t_{i}$, with $1 \leq i \leq n$, the reachability condition $\sigma\left(s_{i}\right) \rightarrow^{*} \sigma\left(t_{i}\right)$ can be proved. We have the following:

Proposition 3. Let $\mathcal{R}$ be a CTRS. A feasibility sequence $\left(s_{i} \bowtie_{i} t_{i}\right)_{i=1}^{n}$ is $\mathcal{R}$ infeasible if $\left(s_{i} \bowtie_{i} t_{i}\right)_{i \in I}$ is $\mathcal{R}$-infeasible for some $I \subseteq\{1, \ldots, n\}$.

Aoto observes the following: for TRSs $\mathcal{R}$, the so-called usable rules for reachability associated to a term $s, \mathcal{U}(\mathcal{R}, s)$ [1, Definition 3], are the only ones we need in any rewriting sequence starting from $s$, i.e., $s \rightarrow_{\mathcal{R}}^{*} t$ iff $s \rightarrow_{\mathcal{U}(\mathcal{R}, s)}^{*} t[1$, Lemma 4]. This also holds for the usable rules $\mathcal{U}(\mathcal{R}, s)$ for CTRSs $\mathcal{R}$ and terms $s$ in $[13$, Definition 11] that we introduce below. First let

$$
\operatorname{RULES}(\mathcal{R}, t) \quad=\quad\left\{\ell \rightarrow r \Leftarrow c \in R \mid \exists p \in \mathcal{P} \operatorname{Pos}(t), \operatorname{root}(\ell)=\operatorname{root}\left(\left.t\right|_{p}\right)\right\}
$$

Note that $R U L E S(\mathcal{R}, t)$ contains the rules of $\mathcal{R}$ which are potentially applicable to the subterms in $t$. Now, the set of usable rules for a term $t$ is:

$$
\mathcal{U}(\mathcal{R}, t)=R U L E S(\mathcal{R}, t) \cup \bigcup_{l \rightarrow r \Leftarrow c \in R U L E S(\mathcal{R}, t)}\left(\mathcal{U}\left(\mathcal{R}^{\sharp}, r\right) \cup \bigcup_{s \approx t \in c} \mathcal{U}\left(\mathcal{R}^{\sharp}, s\right)\right)
$$

where $\mathcal{R}^{\sharp}=\mathcal{R}-\operatorname{RULES}(\mathcal{R}, t)$. That is: we consider both unconditional and conditional rules and add the rules that could be used to evaluate the left-hand 
sides and the conditions when trying to apply those rules to subterms of $t$. Given terms $s_{1}, \ldots, s_{n}$, let $\mathcal{U}\left(\mathcal{R}, s_{1}, \ldots, s_{n}\right)=\bigcup_{i=1}^{n} \mathcal{U}\left(\mathcal{R}, s_{i}\right)$. We have the following.

Proposition 4. Let $\mathcal{R}$ be a CTRS. If $\left(s_{i} \bowtie_{i} t_{i}\right)_{i=1}^{n}$ is $\mathcal{U}\left(\mathcal{R}, s_{1}, \ldots, s_{n}\right)$-feasible, then it is $\mathcal{R}$-feasible. If terms $s_{i}$ are ground for all $1 \leq i \leq n$ and the sequence is $\mathcal{R}$-feasible, then it is $\mathcal{U}\left(\mathcal{R}, s_{1}, \ldots, s_{n}\right)$-feasible.

$\mathcal{R}$-infeasibility of $\left(s_{i} \bowtie_{i} t_{i}\right)_{i=1}^{n}$ can be proved as $\mathcal{U}\left(\mathcal{R}, s_{1}, \ldots, s_{n}\right)$-infeasibility, provided that all terms $s_{i}$ are ground. This requirement cannot be dropped, in general. For $\mathcal{R}=\{\mathrm{a} \rightarrow \mathrm{b}\}$, the sequence $x \rightarrow^{*} \mathrm{a}, x \rightarrow^{*} \mathrm{~b}$ is $\mathcal{R}$-feasible (just instantiate variable $x$ to a). However, it is $\operatorname{not} \mathcal{U}(\mathcal{R}, x, x)$-feasible because $\mathcal{U}(\mathcal{R}, x, x)$ contains no rule.

\section{Use of logical models for proving infeasibility}

In the logic of CTRSs, with binary predicate symbols $\rightarrow$ (for one-step rewriting) and $\rightarrow^{*}$ (for many-step rewriting), the first-order theory $\overline{\mathcal{R}}$ for $\mathcal{R}=(\mathcal{F}, R)$ is obtained from the inference rules in Figure 1 by specializing $(C)_{f, i}$ for each $f \in \mathcal{F}$ and $i, 1 \leq i \leq \operatorname{ar}(f)$ and $(R l)_{\rho}$ for all $\rho: \ell \rightarrow r \Leftarrow c \in R$. Inference rules $\frac{B_{1} \cdots B_{n}}{A}$ become sentences $\left(\forall x_{1}, \ldots, x_{m}\right) B_{1} \wedge \cdots \wedge B_{n} \Rightarrow A$, where $\left\{x_{1}, \ldots, x_{m}\right\}$ is the (possibly empty) set of variables occurring in the atoms $B_{1}, \ldots, B_{n}$ and $A\left[10\right.$, Section 2]. If such a set is empty, we write $B_{1} \wedge \cdots \wedge B_{n} \Rightarrow A$.

Example 5. For $\mathcal{R}$ in Example 1, the associated theory $\overline{\mathcal{R}}$ is:

$$
\begin{array}{cc}
\forall x\left(x \rightarrow^{*} x\right) & (4) \quad \forall x\left(\mathrm{~b} \rightarrow^{*} x \wedge \mathrm{c} \rightarrow^{*} x \Rightarrow \mathrm{a} \rightarrow \mathrm{a}\right) \\
\forall x, y, z\left(x \rightarrow y \wedge y \rightarrow^{*} z \Rightarrow x \rightarrow^{*} z\right)(5) & \forall x\left(\mathrm{~d} \rightarrow^{*} x \wedge \mathrm{e} \rightarrow^{*} x \Rightarrow \mathrm{b} \rightarrow \mathrm{d}\right) \\
& \forall x\left(\mathrm{~d} \rightarrow^{*} x \wedge \mathrm{e} \rightarrow^{*} x \Rightarrow \mathrm{c} \rightarrow \mathrm{d}\right)
\end{array}
$$

Deductions with $\overline{\mathcal{R}}$ proceed $\grave{a}$ la Hilbert by using modus ponens and generalization as inference rules, the usual set of logical axioms, and $\overline{\mathcal{R}}$ as the set of proper axioms [16, Section 2.3]. In this way, we can also prove goals $s \rightarrow t$ and $s \rightarrow^{*} t$, written $\overline{\mathcal{R}} \vdash s \rightarrow t$ and $\overline{\mathcal{R}} \vdash s \rightarrow^{*} t$, respectively. For all terms $s$ and $t$, we have (i) $s \rightarrow_{\mathcal{R}} t$ iff $\overline{\mathcal{R}} \vdash s \rightarrow t$ and (ii) $s \rightarrow_{\mathcal{R}}^{*} t$ iff $\overline{\mathcal{R}} \vdash s \rightarrow^{*} t$.

By a structure $\mathcal{A}$ for a first-order language we mean an interpretation of the function and predicate symbols $(f, g, \ldots$ and $P, Q, \ldots$, respectively) as mappings $f^{\mathcal{A}}, g^{\mathcal{A}}, \ldots$ and relations $P^{\mathcal{A}}, Q^{\mathcal{A}}, \ldots$ on a given set (carrier) also denoted $\mathcal{A}$. Then, the usual interpretation of first-order formulas with respect to the structure is considered. A model for a set $\mathcal{S}$ of first-order sentences (i.e., formulas whose variables are all quantified) is just a structure that makes them all true, written $\mathcal{A} \models \mathcal{S}$. The Herbrand model $\mathcal{H}_{\mathcal{R}}$ for a CTRS $\mathcal{R}$ (or just $\mathcal{H}$ if no confusion arises) consists of the usual Herbrand interpretation with $\mathcal{T}(\mathcal{F})$ as domain, constants interpreted as themselves, i.e., $a^{\mathcal{H}}=a$ for all $s \in \mathcal{F}$ with $\operatorname{ar}(a)=0, k$-ary functions $f \in \mathcal{F}$ interpreted by $f^{\mathcal{H}}\left(t_{1}, \ldots, t_{k}\right)=f\left(t_{1}, \ldots, t_{k}\right)$ for all $t_{1}, \ldots, t_{k} \in \mathcal{T}(\mathcal{F})$ and $\rightarrow^{\mathcal{H}}$ and $\left(\rightarrow^{*}\right)^{\mathcal{H}}$ given by $s \rightarrow^{\mathcal{H}} t$ iff $s \rightarrow_{\mathcal{R}} t$ and $s\left(\rightarrow^{*}\right)^{\mathcal{H}} t$ iff $s \rightarrow_{\mathcal{R}}^{*} t$ for all $s, t \in \mathcal{T}(\mathcal{F})$. By correctness of the first-order 
predicate calculus, for all models $\mathcal{A}$ of a theory $\mathcal{S}$, whenever $\mathcal{S} \vdash \varphi$ holds for a sentence $\varphi$, we have $\mathcal{A}=\varphi$. We use these facts in the following result.

Theorem 6. Let $\mathcal{R}$ be a CTRS, $\left(s_{i} \bowtie_{i} t_{i}\right)_{i=1}^{n}$ be a feasibility sequence, and $\mathcal{A}$ be a structure with nonempty domain. If $\mathcal{A} \models \overline{\mathcal{R}} \cup\left\{\neg(\exists \vec{x}) \bigwedge_{i=1}^{n} s_{i} \bowtie_{i} t_{i}\right\}$, then the sequence is $\mathcal{R}$-infeasible. If $\mathcal{T}(\mathcal{F}) \neq \emptyset$, then $\mathcal{R}$-infeasibility implies that $\mathcal{H}_{\mathcal{R}}=\overline{\mathcal{R}} \cup\left\{\neg(\exists \vec{x}) \bigwedge_{i=1}^{n} s_{i} \bowtie_{i} t_{i}\right\}$ holds.

ProOF. We prove the first claim by contradiction. If $\left(s_{i} \bowtie_{i} t_{i}\right)_{i=1}^{n}$ is $\mathcal{R}$-feasible, then there is a substitution $\sigma$ such that, for all $1 \leq i \leq n, \sigma\left(s_{i}\right) \rightarrow_{\mathcal{R}} \sigma\left(t_{i}\right)$ if $\bowtie_{i}$ is $\rightarrow$ and $\sigma\left(s_{i}\right) \rightarrow_{\mathcal{R}}^{*} \sigma\left(t_{i}\right)$ if $\bowtie_{i}$ is $\rightarrow^{*}$. Since $\mathcal{A}$ is a model of $\overline{\mathcal{R}}$, by correctness we have $\mathcal{A} \models\left(\forall \vec{y}_{i}\right) \sigma\left(s_{i}\right) \bowtie_{i} \sigma\left(t_{i}\right)$ for all $1 \leq i \leq n$, with $\vec{y}_{i}$ the variables in $\operatorname{Var}\left(\sigma\left(s_{i}\right)\right) \cup \mathcal{V} \operatorname{ar}\left(\sigma\left(t_{i}\right)\right)$. Thus, $\mathcal{A} \models(\forall \vec{y})\left(\bigwedge_{i=1}^{n} \sigma\left(s_{i}\right) \bowtie_{i} \sigma\left(t_{i}\right)\right)$, with $\vec{y}$ the variables in $\bigcup_{i=1}^{n} \mathcal{V} \operatorname{ar}\left(\sigma\left(s_{i}\right)\right) \cup \mathcal{V} \operatorname{ar}\left(\sigma\left(t_{i}\right)\right)$. Hence, for all $\nu: \vec{y} \rightarrow$ $\mathcal{A}$, the interpretation in $\mathcal{A}$ of the universally quantified formula above, i.e., $\left[\bigwedge_{i=1}^{n} \sigma\left(s_{i}\right) \bowtie_{i} \sigma\left(t_{i}\right)\right]_{\nu}^{\mathcal{A}}$, is true. Let $\vec{x}$ be the variables in $\bigcup_{i=1}^{n} \mathcal{V} \operatorname{var}\left(s_{i}\right) \cup \mathcal{V} a r\left(t_{i}\right)$. Since the domain of $\mathcal{A}$ is not empty, given an arbitrary valuation $\nu: \vec{y} \rightarrow \mathcal{A}$, there is $\nu^{\prime}: \vec{x} \rightarrow \mathcal{A}$ given by $\nu^{\prime}(x)=[\sigma(x)]_{\nu}^{\mathcal{A}}$ for all variables $x$ in $\vec{x}$, such that $\left[\bigwedge_{i=1}^{n} s_{i} \bowtie_{i} t_{i}\right]_{\nu^{\prime}}^{\mathcal{A}}$ is true. This contradicts the assumption $\mathcal{A} \models \neg(\exists \vec{x})\left(\bigwedge_{i=1}^{n} s_{i} \bowtie_{i}\right.$ $\left.t_{i}\right)$. For the second claim, if $\left(s_{i} \bowtie_{i} t_{i}\right)_{i=1}^{n}$ is $\mathcal{R}$-infeasible, then for all ground substitutions $\sigma$ (which exist due to the assumption $\mathcal{T}(\mathcal{F})=\emptyset$ ) there is $i, 1 \leq$ $i \leq n$ such that $\sigma\left(s_{i}\right) \bowtie \sigma\left(t_{i}\right)$ does not hold. By definition of $\mathcal{H}_{\mathcal{R}}$, and since valuations in $\mathcal{H}_{\mathcal{R}}$ are just ground substitutions (and vice versa), we have that $\mathcal{H}_{\mathcal{R}}=\overline{\mathcal{R}} \cup\left\{\neg(\exists \vec{x}) \bigwedge_{i=1}^{n} s_{i} \bowtie_{i} t_{i}\right\}$ holds.

In order to guarantee that $\mathcal{A}$ in Theorem 6 has a nonempty domain, we just add $(\exists x) x=x$ to $\overline{\mathcal{R}} \cup\{\neg \varphi\}$ (this is not necessary if $\mathcal{T}(\mathcal{F}) \neq \emptyset$ ). Structures $\mathcal{A}$ can be automatically generated from $\overline{\mathcal{R}}$ and sentence $(\mathrm{s}) \neg(\exists \vec{x})\left(\bigwedge_{i=1}^{n} s_{i} \bowtie_{i} t_{i}\right)$ by using tools like AGES [5], implementing the methods in [7]. Essentially, AGES interprets the sort, function and predicate symbols of an input (many-sorted) first-order theory as parametric domains, functions and predicates. Sentences in the theory are then transformed into constraints over such parameters which are then solved by using standard constraint solving methods and tools (based on SAT, SMT, etc.). We also use Mace4 [15], which only computes finite models. Details about the use of AGES and Mace4 to solve the examples in the paper can be found in Appendix A.

Example 7. For $\mathcal{R}$ in Example 1 and $\overline{\mathcal{R}}$ in Example 5, AGES computes a structure $\mathcal{A}$ with domain $\mathbb{N} \cup\{-1\}$ where $\mathrm{a}^{\mathcal{A}}=\mathrm{b}^{\mathcal{A}}=0, \mathrm{c}^{\mathcal{A}}=\mathrm{d}^{\mathcal{A}}=-1$, $\mathrm{e}^{\mathcal{A}}=1, x \rightarrow^{\mathcal{A}} y$ iff $x=y=-1$ and $x\left(\rightarrow^{*}\right)^{\mathcal{A}} y$ iff $x=y$. This is a model of $\overline{\mathcal{R}} \cup\left\{\neg(\exists x)\left(\mathrm{b} \rightarrow^{*} x \wedge \mathrm{c} \rightarrow^{*} x\right), \neg(\exists x)\left(\mathrm{d} \rightarrow^{*} x \wedge \mathrm{e} \rightarrow^{*} x\right)\right\}$. By Theorem 6, both $\mathrm{b} \rightarrow^{*} x, \mathrm{c} \rightarrow^{*} x$ and $\mathrm{d} \rightarrow^{*} x, \mathrm{e} \rightarrow^{*} x$ are $\mathcal{R}$-infeasible.

\section{Applications}

In this section we explore the use of Theorem 6 in the application areas (i)-(vii) in the introduction. We mostly consider examples from the literature. 


\subsection{Infeasible rules and conditional dependency pairs}

Rules $\ell \rightarrow r \Leftarrow c$ with a conditional part $c$ whose associated sequence $s_{1} \rightarrow^{*}$ $t_{1}, \ldots, s_{n} \rightarrow^{*} t_{n}$ is $\mathcal{R}$-infeasible are called infeasible rules. Example 7 shows that all rules of $\mathcal{R}$ in Example 1 are infeasible.

A CTRS $\mathcal{R}$ is operationally terminating iff no term $t$ has an infinite proof tree using the inference system in Figure 1 [9]. As for TRSs [2], the dependency pair approach provides a suitable way to mechanize proofs of operational termination of CTRSs $[12,14]$. Dependency pairs are just conditional rules $u \rightarrow v \Leftarrow c$ which are obtained from the CTRS $\mathcal{R}$. Proving infeasibility of such rules is also useful to prove operational termination of CTRSs, see [14].

\subsection{Infeasible critical pairs}

A conditional critical pair (CCP) $\langle s, t\rangle \Leftarrow \sigma(c), \sigma\left(c^{\prime}\right)$ is obtained from (renamed versions of) rules $\ell \rightarrow r \Leftarrow c$ and $\ell^{\prime} \rightarrow r^{\prime} \Leftarrow c^{\prime}$ iff there is a nonvariable subterm $t$ of $\ell$ such that $\sigma(\ell)=\sigma(t)$ for a most general unifier $\sigma$ [19, Definition 7.1.8]. A critical pair $\langle s, t\rangle \Leftarrow s_{1} \approx t_{1}, \ldots, s_{n} \approx t_{n}$ is infeasible if $\left(s_{i} \rightarrow^{*} t_{i}\right)_{i=1}^{n}$ is $\mathcal{R}$-infeasible. Joinability of feasible CCPs can be used to prove confluence of CTRSs [19, Section 7.3]. Infeasible CCPs can be dismissed, though.

Example 8. The following CTRS $\mathcal{R}$ [21, Example 23]:

$$
\mathrm{g}(x) \rightarrow \mathrm{f}(x, x) \quad(9) \quad \mathrm{g}(x) \rightarrow \mathrm{g}(x) \Leftarrow \mathrm{g}(x) \approx \mathrm{f}(\mathrm{a}, \mathrm{b})
$$

has a conditional critical pair $\langle\mathrm{f}(x, x), \mathrm{g}(x)\rangle \Leftarrow \mathrm{g}(x) \approx \mathrm{f}(\mathrm{a}, \mathrm{b})$. With AGES, we obtain a model of $\overline{\mathcal{R}} \cup\left\{\neg(\exists x) \mathrm{g}(x) \rightarrow^{*} \mathrm{f}(\mathrm{a}, \mathrm{b})\right\}$ which proves infeasibility of the conditional critical pair. In [21, Example 23] this is proved by using unification tests together with a transformation. It is discussed that the alternative tree automata techniques investigated in the paper do not work for this example.

\subsection{Root-stability and irreducibility}

A term $t$ is root-stable (with respect to a TRS $\mathcal{R}$ ) if $t$ cannot be reduced to a redex, i.e., there is no rule $\ell \rightarrow r \in \mathcal{R}$ such that $t \rightarrow^{*} \sigma(\ell)$ for some substitution

$\sigma$ [18]. Root-stability is important in the semantic description of lazy languages where infinite normal forms are allowed and approximated as sequences of rootstable terms [18]. Clearly, we can prove a ground term $t$ root-stable if we (often independently) show $\mathcal{R}$-infeasibility of $t \rightarrow^{*} \ell$ for all $\ell \rightarrow r \in \mathcal{R}$.

Example 9. Consider the following TRS $\mathcal{R}$ :

$$
\begin{aligned}
& \mathrm{a} \rightarrow \mathrm{b} \\
& \mathrm{b} \rightarrow \mathrm{a}
\end{aligned}
$$

$$
\mathrm{f}(x, x) \rightarrow \mathrm{c}
$$

We prove root-stability of $\mathrm{f}(\mathrm{a}, \mathrm{c})$ by showing $\mathcal{R}$-infeasibility of $\mathrm{f}(\mathrm{a}, \mathrm{c}) \rightarrow^{*} \mathrm{f}(x, x)$, $\mathrm{f}(\mathrm{a}, \mathrm{c}) \rightarrow^{*} \mathrm{a}$, and $\mathrm{f}(\mathrm{a}, \mathrm{c}) \rightarrow^{*} \mathrm{~b}$. With Mace4 we obtain a model of

$$
\overline{\mathcal{R}} \cup\left\{\neg(\exists x) \mathrm{f}(\mathrm{a}, \mathrm{c}) \rightarrow^{*} \mathrm{f}(x, x), \neg\left(\mathrm{f}(\mathrm{a}, \mathrm{c}) \rightarrow^{*} \mathrm{a}\right), \neg\left(\mathrm{f}(\mathrm{a}, \mathrm{c}) \rightarrow^{*} \mathrm{~b}\right)\right\}
$$


A term $t$ is irreducible if no rewriting step is possible on it. For ground terms, we can prove irreducibility of $t$ as the $\mathcal{R}$-infeasibility of $(\exists x) t \rightarrow x$.

Example 10. Consider the following CTRS $\mathcal{R}$ [13, Example 3]

$$
\begin{aligned}
\mathrm{g}(\mathrm{a}) & \rightarrow \mathrm{c}(\mathrm{b}) \\
\mathrm{b} & \rightarrow \mathrm{f}(\mathrm{a})
\end{aligned}
$$

$$
\mathrm{f}(x) \quad \rightarrow \quad x \Leftarrow \mathrm{g}(x) \rightarrow \mathrm{c}(y)
$$

Mace4 obtains a model of $\overline{\mathcal{R}} \cup\{\neg(\exists x) \mathrm{f}(\mathrm{c}(\mathrm{a})) \rightarrow x\}$, i.e., $\mathrm{f}(\mathrm{c}(\mathrm{a}))$ is irreducible.

\subsection{Non-joinable terms}

We can see the usual joinability problem $s \downarrow t$, i.e., the existence of a term $u$ such that $s \rightarrow_{\mathcal{R}}^{*} u$ and $t \rightarrow_{\mathcal{R}}^{*} u$, as a specific feasibility sequence.

Theorem 11. Let $\mathcal{R}$ be a CTRS, $s, t$ be terms, and $x$ be a fresh variable not occurring in $s$ or $t$. If $s$ and $t$ are joinable, then $s \rightarrow^{*} x, t \rightarrow^{*} x$ is $\mathcal{R}$-feasible. If $s$ and $t$ are ground and $s \rightarrow^{*} x, t \rightarrow^{*} x$ is $\mathcal{R}$-feasible, then $s$ and $t$ are joinable.

Proof. If $s$ and $t$ are joinable, then $s \rightarrow_{\mathcal{R}}^{*} u$ and $t \rightarrow_{\mathcal{R}}^{*} u$ for some term $u$. Hence, $s \rightarrow^{*} x, t \rightarrow^{*} x$ is $\mathcal{R}$-feasible (use $\sigma$ such that $\sigma(x)=u$ and $\sigma(y)=y$ for any other variable $y \neq x)$. On the other hand, if $s$ and $t$ are ground and $s \rightarrow^{*} x, t \rightarrow^{*} x$ is $\mathcal{R}$-feasible, then there is a substitution $\sigma$ such that $\sigma(s)=$ $s \rightarrow_{\mathcal{R}}^{*} u=\sigma(x)$ and $\sigma(t)=t \rightarrow_{\mathcal{R}}^{*} u=\sigma(x)$, i.e., $s$ and $t$ are joinable.

By Theorem 11, $\mathcal{R}$-infeasibility of $s \rightarrow^{*} x, t \rightarrow^{*} x$ implies non-joinability of $s$ and $t$ (where $s$ and $t$ may contain variables).

Example 12. Consider the following CTRS $\mathcal{R}$ [19, Example 7.3.3]:

$$
\mathrm{a} \rightarrow \mathrm{b}
$$

$$
\mathrm{f}(x) \rightarrow \mathrm{c} \Leftarrow x \approx \mathrm{a}
$$

There is no CCP but $\mathcal{R}$ is not confluent: $\mathrm{f}(\mathrm{a}) \rightarrow_{\mathcal{R}} \mathrm{f}(\mathrm{b})$ and $\mathrm{f}(\mathrm{a}) \rightarrow_{\mathcal{R}} \mathrm{c}$ but $\mathrm{c}$ and $\mathrm{f}(\mathrm{b})$ are not joinable. With AGES we obtain a model of $\overline{\mathcal{U}(\mathcal{R}, \mathrm{f}(\mathrm{b}), \mathrm{c})} \cup$ $\left\{\neg(\exists x)\left(\mathrm{f}(\mathrm{b}) \rightarrow^{*} x \wedge \mathrm{c} \rightarrow^{*} x\right)\right\}$, where $\mathcal{U}(\mathcal{R}, \mathrm{f}(\mathrm{b}), \mathrm{c})=\{(18)\}$ (see Proposition 4). Therefore, $\mathrm{f}(\mathrm{b})$ and $\mathrm{c}$ are proved non-joinable.

\subsection{Approximation of dependency graphs}

In the dependency pair approach for termination analysis of TRSs $\mathcal{R}$, the notion of chain of dependency pairs is paramount [2]: a sequence of rules $u_{1} \rightarrow$ $v_{1}, u_{2} \rightarrow v_{2}, \ldots$ (called dependency pairs) is said to be a chain if there is a substitution $\sigma$ such that $\sigma\left(v_{i}\right) \rightarrow_{\mathcal{R}}^{*} \sigma\left(u_{i+1}\right)$ for all $i \geq 1$. Termination of TRSs is characterized as the absence of infinite chains of dependency pairs. For finite TRSs, infinite chains can always be represented as cycles in a dependency graph whose nodes are dependency pairs; there is an arc from a 'node' $u \rightarrow v$ to another node $u^{\prime} \rightarrow v^{\prime}$ (previously renamed, to share no variable with $u \rightarrow v$ ) if there is a substitution $\sigma$ such that $\sigma(v) \rightarrow_{\mathcal{R}}^{*} \sigma\left(u^{\prime}\right)$. Thus, the $\mathcal{R}$-infeasibility of $v \rightarrow^{*} u^{\prime}$ implies the absence of an arc from $u \rightarrow v$ to $u^{\prime} \rightarrow v^{\prime}$. The dependency graph is 
not computable due to the undecidability of the involved reachability problems. Several overapproximations are available [2, 4]. However, avoiding 'false' arcs is essential to obtain more efficient termination proofs: we pay attention to dependency pairs in cycles of the graph only.

Example 13. Consider the following TRS [17, Example 21]:

$$
\begin{aligned}
& \mathrm{f}(x, \mathrm{a}) \rightarrow \mathrm{f}(x, \mathrm{~g}(x, \mathrm{~b})) \\
& \mathrm{g}(\mathrm{a}, y) \rightarrow y
\end{aligned}
$$

There are three dependency pairs:

$$
\begin{aligned}
& \mathrm{F}(x, \mathrm{a}) \rightarrow \mathrm{F}(x, \mathrm{~g}(x, \mathrm{~b})) \\
& \mathrm{F}(x, \mathrm{a}) \rightarrow \mathrm{G}(x, \mathrm{~b})
\end{aligned}
$$

$$
\mathrm{g}(\mathrm{h}(x), y) \rightarrow \mathrm{g}(x, \mathrm{~h}(y))
$$

The estimated graph which is obtained by using [4] (usually implemented in termination tools) is the following:

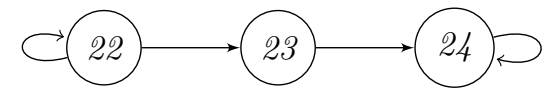

Middeldorp discusses several refinements that use tree automata techniques for the approximation of the dependency graph. By using one of them (the socalled nv approximation [17, Definition 8]) he obtains the graph above. By using a second one, he shows that the arcs from (22) to itself and from (22) to (23) can be removed safely, as they do not belong to the dependency graph (there is no substitution such that $\sigma(\mathrm{F}(x, \mathrm{~g}(x, \mathrm{~b}))) \rightarrow_{\mathcal{R}}^{*} \sigma(\mathrm{F}(y, \mathrm{a}))$, note the renaming introduced in the second term). With AGES we obtain a model of $\overline{\mathcal{R}} \cup\left\{\neg(\exists x) \mathrm{F}(x, \mathrm{~g}(x, \mathrm{~b})) \rightarrow^{*} \mathrm{~F}(y, \mathrm{a})\right\}$ which shows that these two arcs do not belong to the dependency graph.

\section{Conclusions}

We have presented a semantic approach to prove infeasibility in term rewriting: in order to prove the infeasibility of a sequence $\left(s_{i} \bowtie_{i} t_{i}\right)_{i=1}^{n}$ of rewriting goals, we try to find a model of a set $\overline{\mathcal{R}}$ of first-order sentences representing the operational semantics of $\mathcal{R}$ together with a sentence $\neg(\exists \vec{x}) \bigwedge_{i=1}^{n} s_{i} \bowtie_{i} t_{i}$.

We have shown the usefulness of this approach by an exhaustive consideration of examples from several recent papers addressing the role of infeasibility when applied to such problems. In particular, we could deal with all of Aoto's examples in [1] (regarding proofs of non-joinability of ground terms in TRSs), see Appendix B. In [17], Middeldorp uses different approximations to the estimation of the dependency graph for TRSs in the analysis of termination using dependency pairs. We were also able to deal with all examples reported in [17] (see Example 13 and Appendix C). We also dealt with all examples solved in [20] (see Appendix D), and [21] (see Example 8 and Appendix E). Note that these papers explore several alternative methods and, as reported by the authors, some of them fail in specific examples which require a different approach. 
In contrast to all aforementioned works, we are using a single technique to deal with all kinds of reported problems. Of course, we do not claim to actually outperform them. However, in view of these good results, we believe that the technique is promising.

We do not have a dedicated, fully automated 'infeasibility' checker yet. Instead, we just encode the problem we want to deal with as a specific infeasibility sequence and then use AGES or Mace4 to apply Theorem 6. In AGES, the generation of a single model is completely automatic, but in order to succeed on a given problem, we may need to try different configurations for the generation of several models. In contrast to Mace4, whose generation scheme is simpler and where this is automatic, AGES currently requires user assistance to change settings like the dimension of the domains or the use of piecewise interpretations (like in Example 8), which are usually 'expensive' and not part of the default configuration. Thus, further investigation developing heuristics and strategies for an efficient use of the technique is envisaged as a subject for future work.

Acknowledgments. We thank the anonymous referees for many remarks and suggestions that led to improve the paper.

\section{References}

[1] T. Aoto. Disproving Confluence of Term Rewriting Systems by Interpretation and Ordering. In P. Fontaine, C. Ringeissen, and R.A. Schmidt, editors, Proc. of the 9th International Symposium on Frontiers of Combining Systems, FroCoS'13, LNCS 8152:311-326, 2013.

[2] T. Arts and J. Giesl. Termination of Term Rewriting Using Dependency Pairs. Theoretical Computer Science, 236(1-2):133-178, 2000.

[3] F. Baader and T. Nipkow. Term Rewriting and All That. Cambridge University Press, 1998.

[4] J. Giesl, R. Thiemann, and P. Schneider-Kamp. Proving and Disproving Termination of Higher-Order Functions. In B. Gramlich, editor, Proc. of 5th International Workshop on Frontiers of Combining Systems, FroCoS'05, LNAI 3717:216-231, 2005.

[5] R. Gutiérrez, S. Lucas, and P. Reinoso. A tool for the automatic generation of logical models of order-sorted first-order theories. In A. Villanueva, editor, Proc. of the XVI Jornadas sobre Programación y Lenguajes, PROLE'16, pages 215-230, 2016. http://hdl.handle.net/11705/PROLE/2016/018. Tool available at http://zenon.dsic.upv.es/ages/.

[6] S. Lucas. Analysis of Rewriting-Based Systems as First-Order Theories. In F. Fioravanti and J.P. Gallagher, editors, Revised Selected papers from the 27th International Symposium on Logic-Based Program Synthesis and Transformation, LOPSTR 2017, LNCS to appear, 2018.

[7] S. Lucas and R. Gutiérrez. Automatic Synthesis of Logical Models for OrderSorted First-Order Theories. Journal of Automated Reasoning, 60(4):465-501, 2018. 
[8] S. Lucas and R. Gutiérrez. A Semantic Criterion For Proving Infeasibitlity In Conditional Rewriting. In B. Accatoli and B. Felgenhauer, editors, Proc. of the 6th International Workshop on Confluence, IWC 2017, pages 15-19, 2017.

[9] S. Lucas, C. Marché, and J. Meseguer. Operational termination of conditional term rewriting systems. Information Processing Letters, 95:446-453, 2005.

[10] S. Lucas and J. Meseguer. Models for Logics and Conditional Constraints in Automated Proofs of Termination. In G.A. Aranda-Corral, J. Calmet, and F.J. Martín-Mateos, editors, Proc. of the 12th International Conference on Artificial Intelligence and Symbolic Computation, AISC'14, LNAI 8884:9-20, 2014.

[11] S. Lucas and J. Meseguer. 2D Dependency Pairs for Proving Operational Termination of CTRSs. In S. Escobar, editor, Proc. of the 10th International Workshop on Rewriting Logic and its Applications, WRLA'14, LNCS 8663:195-212, 2014.

[12] S. Lucas and J. Meseguer. Dependency pairs for proving termination properties of conditional term rewriting systems. Journal of Logical and Algebraic Methods in Programming, 86:236-268, 2017.

[13] S. Lucas and J. Meseguer. Normal forms and normal theories in conditional rewriting. Journal of Logical and Algebraic Methods in Programming, 85(1):67-97, 2016.

[14] S. Lucas, J. Meseguer, and R. Gutiérrez. Extending the 2D DP Framework for Conditional Term Rewriting Systems. In M. Proietti and H. Seki, editors, Selected papers of the 24th International Symposium on Logic-Based Program Synthesis and Transformation LOPSTR'14, LNCS 8981:113-130, 2015.

[15] W. McCune Prover9 and Mace4. http://www.cs.unm.edu/ mccune/prover9/, 2005-2010.

[16] E. Mendelson. Introduction to Mathematical Logic. Fourth edition. Chapman \& Hall, 1997.

[17] A. Middeldorp. Approximating Dependency Graphs Using Tree Automata Techniques. In R. Goré, A. Leitsch, and T. Nipkow, editors, Proc. of the First International Joint Conference on Automated Reasoning, IJCAR'01, LNAI 2083:593610, 2001.

[18] A. Middeldorp. Call by Need Computations to Root-Stable Form. In Proc. of the 24th ACM SIGPLAN-SIGACT symposium on Principles of Programming Languages, POPL'97, pages 94-105. ACM, 1997.

[19] E. Ohlebusch. Advanced Topics in Term Rewriting. Springer-Verlag, Apr. 2002.

[20] T. Sternagel and A. Middeldorp. Infeasible Conditional Critical Pairs. In A. Tiwari and T. Aoto, editors, Proc. of the 4 th International Workshop on Confluence, IWC'15, pages 13-18, 2014.

[21] C. Sternagel and T. Sternagel. Certifying Confluence Of Almost Orthogonal CTRSs Via Exact Tree Automata Completion. In D. Kesner and B. Pientka, editors, Proc. of the 1st International Conference on Formal Structures for Computation and Deduction, FSCD'16, LIPIcs 52, Article No. 85; pp. 85:1-85:16, 2016. 
Appendix A. Generation of models for the examples in the paper with AGES and Mace4

\section{Example 7: infeasible rules}

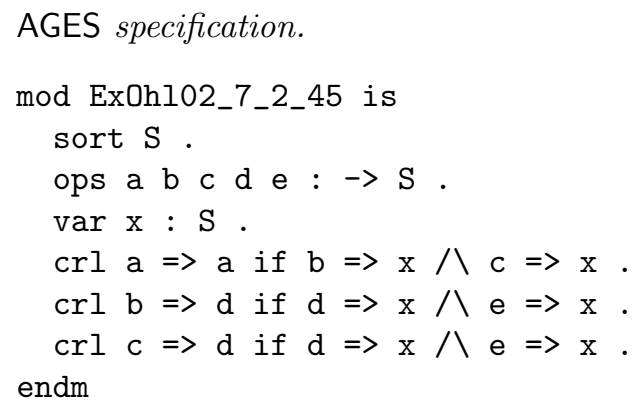

AGES goal. The current version of AGES does not accept explicit quantification in goals; universal quantification is assumed. Thus, $\neg(\exists \vec{x})\left(s_{1} \rightarrow^{*} t_{1} \wedge \cdots \wedge\right.$ $\left.s_{n} \rightarrow^{*} t_{n}\right)$ must be introduced as $\neg\left(s_{1} \rightarrow^{*} t_{1} \wedge \cdots \wedge s_{n} \rightarrow^{*} t_{n}\right)$, which actually corresponds to the equivalent universally quantified version $(\forall \vec{x}) \neg\left(s_{1} \rightarrow^{*} t_{1} \wedge\right.$ $\cdots \wedge s_{n} \rightarrow^{*} t_{n}$ ) of the original sentence.

$\sim(\mathrm{b}->* \mathrm{x}: \mathrm{S} / \mathrm{c}->* \mathrm{x}: \mathrm{S})$

$\sim(\mathrm{d}->* \mathrm{x}: \mathrm{S} / \mathrm{e}->* \mathrm{x}: \mathrm{S})$

AGES output.

Domains :

$\mathrm{S}: \mid \mathrm{N} \cup\{-1\}$

Function Interpretations:

$|[a]|=0$

$|[\mathrm{b}]|=0$

$|[\mathrm{c}]|=-1$

$|[\mathrm{d}]|=-1$

$|[e]|=1$

Predicate Interpretations:

$\mathrm{x}_{-} 1 \_1: \mathrm{S} \rightarrow * \mathrm{x}_{-} 2 \_1: \mathrm{S} \Leftrightarrow\left(\left(\mathrm{x}_{-} 1 \_1: \mathrm{S}>=\mathrm{x}_{-} 2 \_1: \mathrm{S}\right)\right.$ 八 ( $\left.\left.\mathrm{x}_{-} 2 \_1: \mathrm{S}>=\mathrm{x}_{-} 1 \_1: \mathrm{S}\right)\right)$

$\mathrm{x}_{-} 1 \_1: \mathrm{S} \rightarrow \mathrm{x}_{-} 2 \_1: \mathrm{S} \Leftrightarrow\left(\left(0>=2+2 . \mathrm{x}_{-}{ }_{2} \_1: \mathrm{S}\right) 八\left(\mathrm{x}_{-} 2 \_1: \mathrm{S}>=\mathrm{x}_{-} 1 \_1: \mathrm{S}\right)\right)$

Computed model. The computed structure $\mathcal{A}$ has domain $\mathbb{N} \cup\{-1\}$. Symbols are interpreted as follows:

$$
\begin{array}{rlrl}
\mathrm{a}^{\mathcal{A}} & =\mathrm{b}^{\mathcal{A}}=0 & \mathrm{c}^{\mathcal{A}}=\mathrm{d}^{\mathcal{A}}=-1 & \mathrm{e}^{\mathcal{A}}=1 \\
x & \rightarrow{ }^{\mathcal{A}} y \Leftrightarrow x=y=-1 & x\left(\rightarrow^{*}\right)^{\mathcal{A}} y \Leftrightarrow x=y &
\end{array}
$$




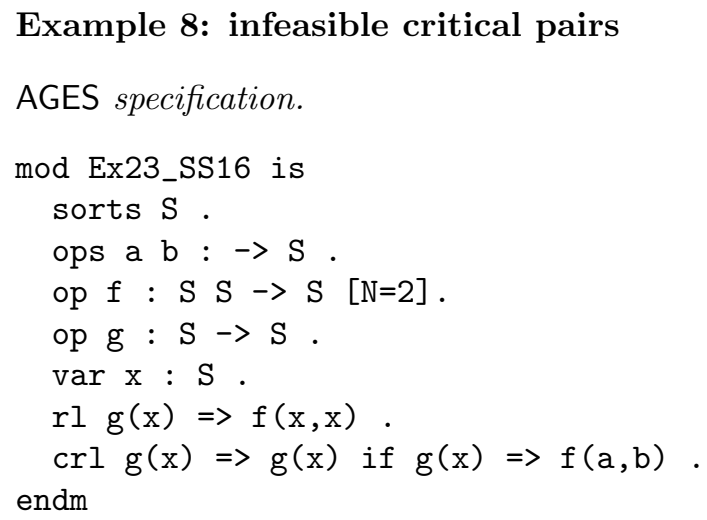

AGES goal.

$\sim(g(x: S)->* f(a, b))$

AGES output.

Domains :

$\mathrm{S}: \mid \mathrm{N} \cup\{-1\}$

Function Interpretations:

$\left|\left[g\left(x_{-} 1_{-} 1: S\right)\right]\right|=1+x_{-} 1_{-} 1: S$

$|[a]|=0$

$|[b]|=-1$

$\left|\left[f\left(x_{-} 1_{-} 1: S, x_{-} 2 \_1: S\right)\right]\right|=$

$1+\mathrm{x}_{-}{ }_{-} 1: \mathrm{S}$ if $\left(\mathrm{x}_{-}{ }_{2} \_1: \mathrm{S}>=\mathrm{x}_{-}{ }_{-} 1: \mathrm{S}\right)$

$-1+x_{-} 1 \_1: S$ otherwise

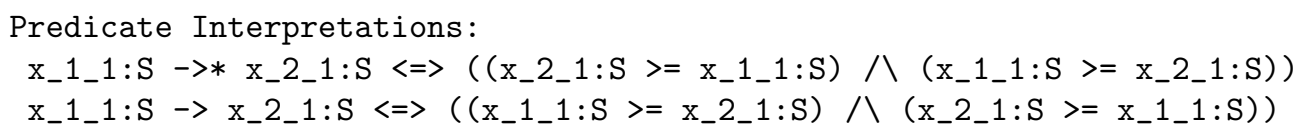

Computed model. The computed structure $\mathcal{A}$ has domain $\mathbb{N} \cup\{-1\}$. Symbols are interpreted as follows:

$$
\begin{aligned}
& \mathrm{a}^{\mathcal{A}}=0 \quad \mathrm{~b}^{\mathcal{A}}=-1 \quad \mathrm{f}^{\mathcal{A}}(x, y)= \begin{cases}x+1 & \text { if } y \geq x \\
x-1 & \text { otherwise }\end{cases} \\
& \mathrm{g}^{\mathcal{A}}(x)=x+1 \quad x \rightarrow^{\mathcal{A}} y \Leftrightarrow x=y \quad x\left(\rightarrow^{*}\right)^{\mathcal{A}} y \Leftrightarrow x=y
\end{aligned}
$$




\section{Example 9: root-stability}

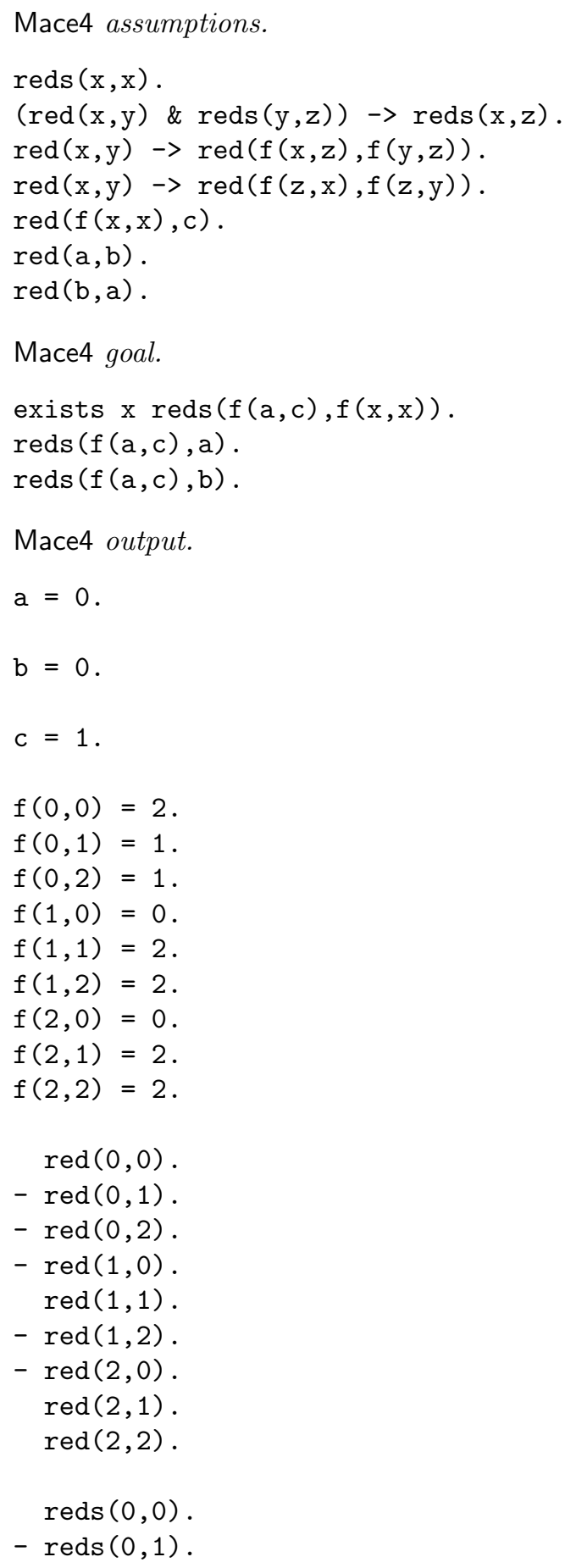


$-\operatorname{reds}(0,2)$.

$-\operatorname{reds}(1,0)$.

$\operatorname{reds}(1,1)$.

$-\operatorname{reds}(1,2)$.

- reds $(2,0)$. reds $(2,1)$. $\operatorname{reds}(2,2)$.

Computed model. The computed structure $\mathcal{A}$ has domain $\mathbb{N} \cup\{0,1,2\}$. Symbols are interpreted as follows:

$$
\begin{aligned}
& \mathrm{a}^{\mathcal{A}}=\mathrm{b}^{\mathcal{A}}=0 \mathrm{f}^{\mathcal{A}}(x, y)= \begin{cases}2 & \text { if } x, y \geq 1 \vee x=y=0 \\
1 & \text { if } x=0 \wedge y \neq 0 \\
0 & \text { otherwise }\end{cases} \\
& \mathrm{c}^{\mathcal{A}}=1 \quad x \rightarrow^{\mathcal{A}} y \Leftrightarrow x\left(\rightarrow^{*}\right)^{\mathcal{A}} y \Leftrightarrow x=y \vee(x=2 \wedge y=1)
\end{aligned}
$$




\section{Example 10: irreducibility}

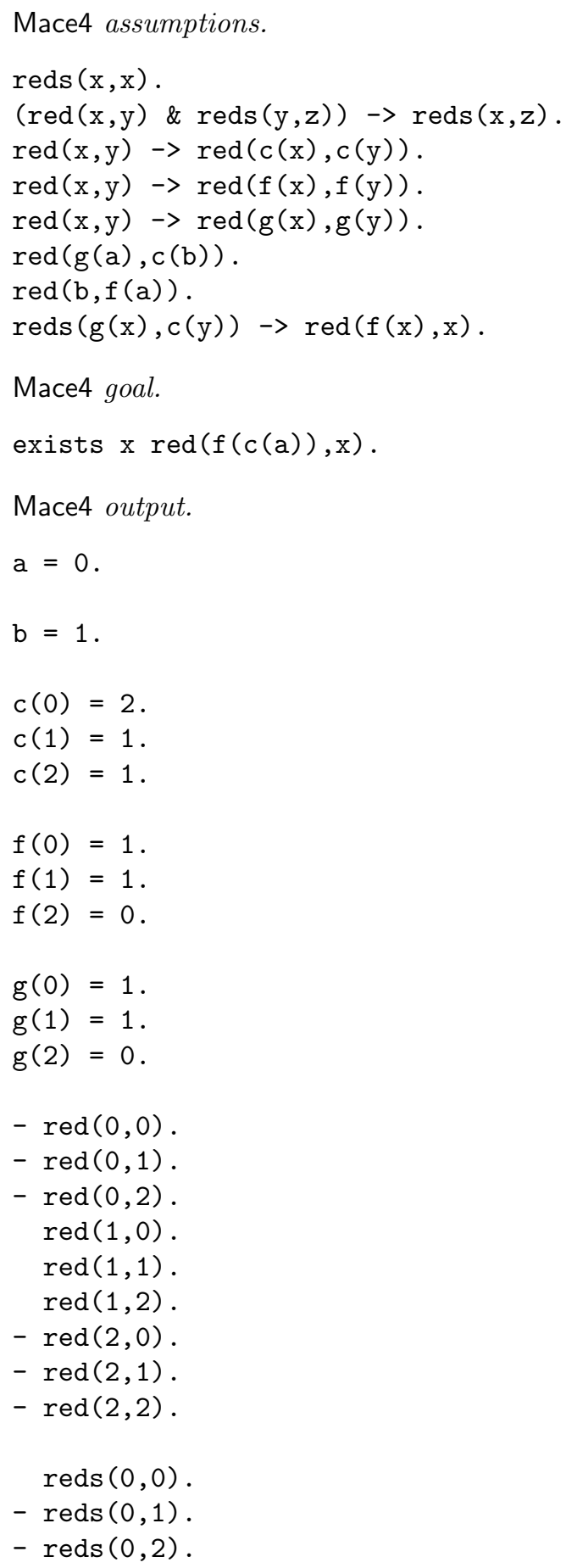




$$
\begin{array}{r}
\operatorname{reds}(1,0) . \\
\operatorname{reds}(1,1) . \\
\operatorname{reds}(1,2) . \\
-\operatorname{reds}(2,0) . \\
-\operatorname{reds}(2,1) . \\
\operatorname{reds}(2,2) .
\end{array}
$$

Computed model. The computed structure $\mathcal{A}$ has domain $\{0,1,2\}$. Symbols are interpreted as follows:

$$
\begin{aligned}
& \mathrm{a}^{\mathcal{A}}=0 \quad \mathrm{c}^{\mathcal{A}}(x)= \begin{cases}2 & \text { if } x=0 \\
1 & \text { otherwise }\end{cases} \\
& \mathrm{b}^{\mathcal{A}}=1 \quad x \rightarrow^{\mathcal{A}} y \Leftrightarrow x=1 \\
& \mathrm{f}^{\mathcal{A}}(x)=\mathrm{g}^{\mathcal{A}}(x)= \begin{cases}0 & \text { if } x=2 \\
1 & \text { otherwise }\end{cases} \\
& x\left(\rightarrow^{*}\right)^{\mathcal{A}} y \Leftrightarrow x=y \vee x=1
\end{aligned}
$$




\section{Example 12: non-joinable terms}

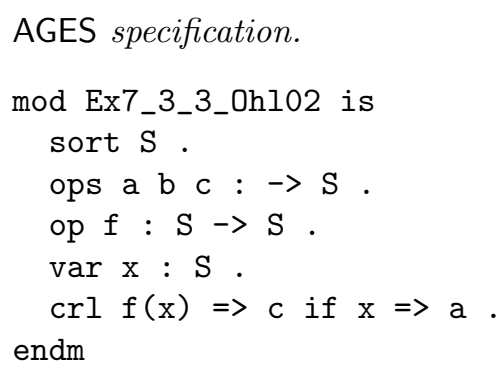

AGES output.

Domains :

S: $\mid N \cup\{-1\}$

Function Interpretations:

$\left|\left[f\left(x_{-} 1 \_1: S\right)\right]\right|=x_{-} 1 \_1: S$

$|[a]|=0$

$|[\mathrm{b}]|=-1$

$|[c]|=0$

Predicate Interpretations:

$\mathrm{x}_{-} 1 \_1: \mathrm{S} \rightarrow * \mathrm{x}_{-} 2 \_1: \mathrm{S} \Leftrightarrow\left(\left(\mathrm{x}_{-} 2_{-} 1: \mathrm{S}>=\mathrm{x}_{-} 1 \_1: \mathrm{S}\right) 八\left(\mathrm{x}_{-} 1 \_1: \mathrm{S}>=\mathrm{x}_{-}{ }_{2} \_1: \mathrm{S}\right)\right)$

$\mathrm{x}_{-} 1 \_1: \mathrm{S} \rightarrow \mathrm{x}_{-} 2 \_1: \mathrm{S} \Leftrightarrow\left(\left(\mathrm{x}_{-} 2 \_1: \mathrm{S}>=\mathrm{x}_{-} 1 \_1: \mathrm{S}\right) 八\left(\mathrm{x}_{-} 1 \_1: \mathrm{S}>=\mathrm{x}_{-} 2 \_1: \mathrm{S}\right)\right)$

Computed model. The computed structure $\mathcal{A}$ has domain $\mathbb{N} \cup\{-1\}$. Symbols are interpreted as follows:

$$
\begin{array}{rlrlrl}
\mathrm{a}^{\mathcal{A}} & =0 & \mathrm{~b}^{\mathcal{A}}=-1 & \mathrm{c}^{\mathcal{A}}=0 & \mathrm{f}^{\mathcal{A}}(x)=x \\
x \rightarrow{ }^{\mathcal{A}} y & \Leftrightarrow x=y & x\left(\rightarrow^{*}\right)^{\mathcal{A}} y \Leftrightarrow x=y & &
\end{array}
$$




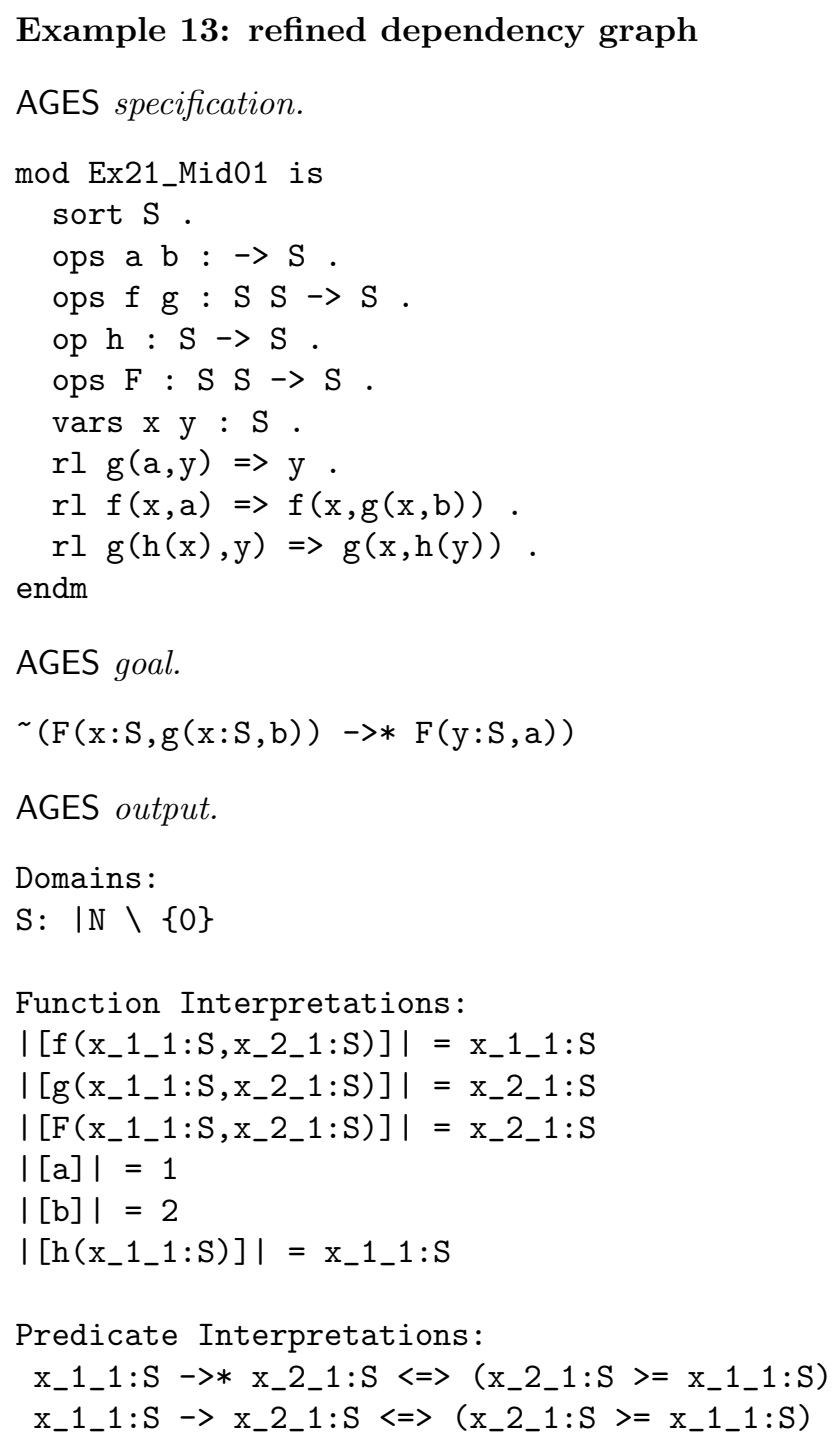

Computed model. The computed structure $\mathcal{A}$ has domain $\mathbb{N}-\{0\}$. Symbols are interpreted as follows:

$$
\begin{array}{rlrlrl}
\mathrm{a}^{\mathcal{A}}=1 & \mathrm{~b}^{\mathcal{A}}=2 & \mathrm{f}^{\mathcal{A}}(x, y)=x & \mathrm{~g}^{\mathcal{A}}(x, y) & =y \\
\mathrm{~h}^{\mathcal{A}}(x)=x & \mathrm{~F}^{\mathcal{A}}(x, y)=y & x \rightarrow^{\mathcal{A}} y \Leftrightarrow y \geq x & x\left(\rightarrow^{*}\right)^{\mathcal{A}} y \Leftrightarrow y \geq x
\end{array}
$$




\section{Appendix B. Additional examples from [1]}

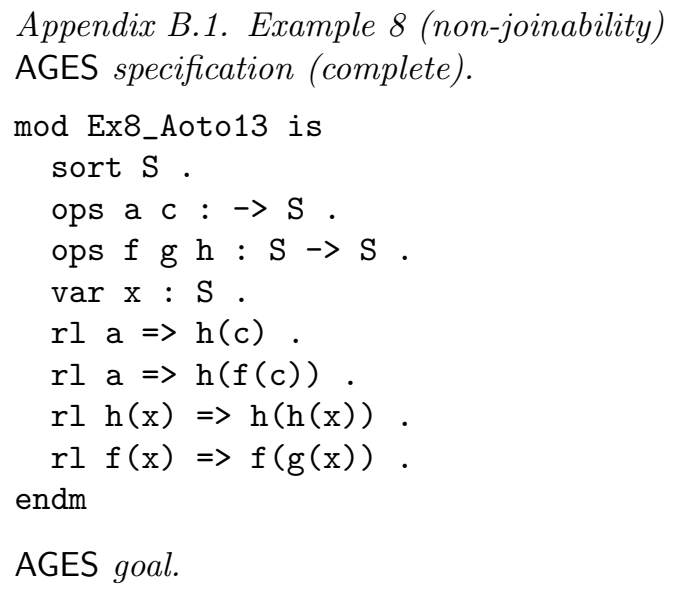

We restrict the attention to the usable rules (Proposition 4):

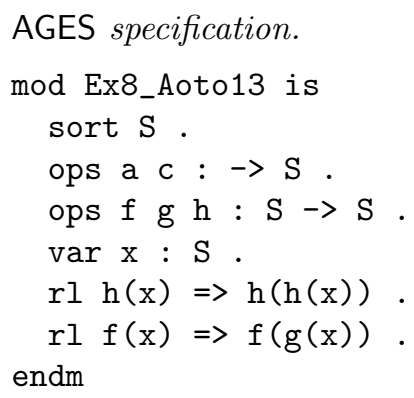

Computed model. The computed structure $\mathcal{A}$ has domain $\{0,1\}$. Symbols are interpreted as shown above. 


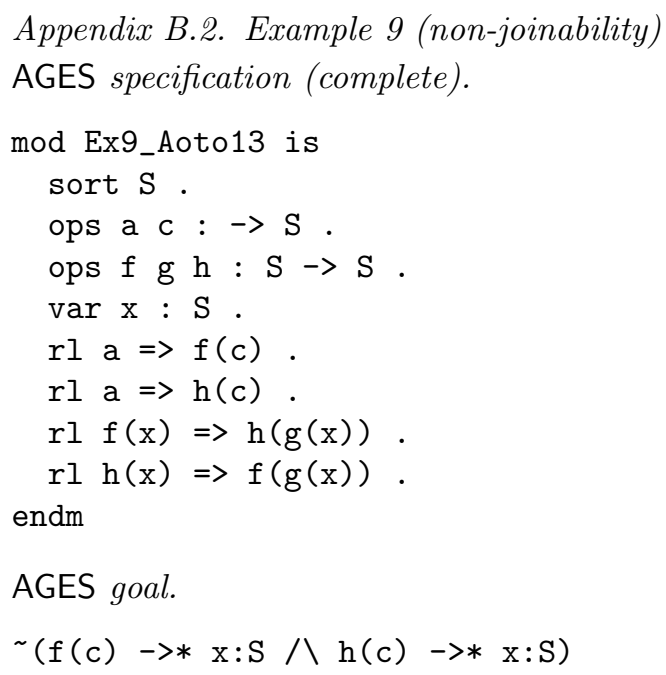

We restrict the attention to the usable rules (Proposition 4):

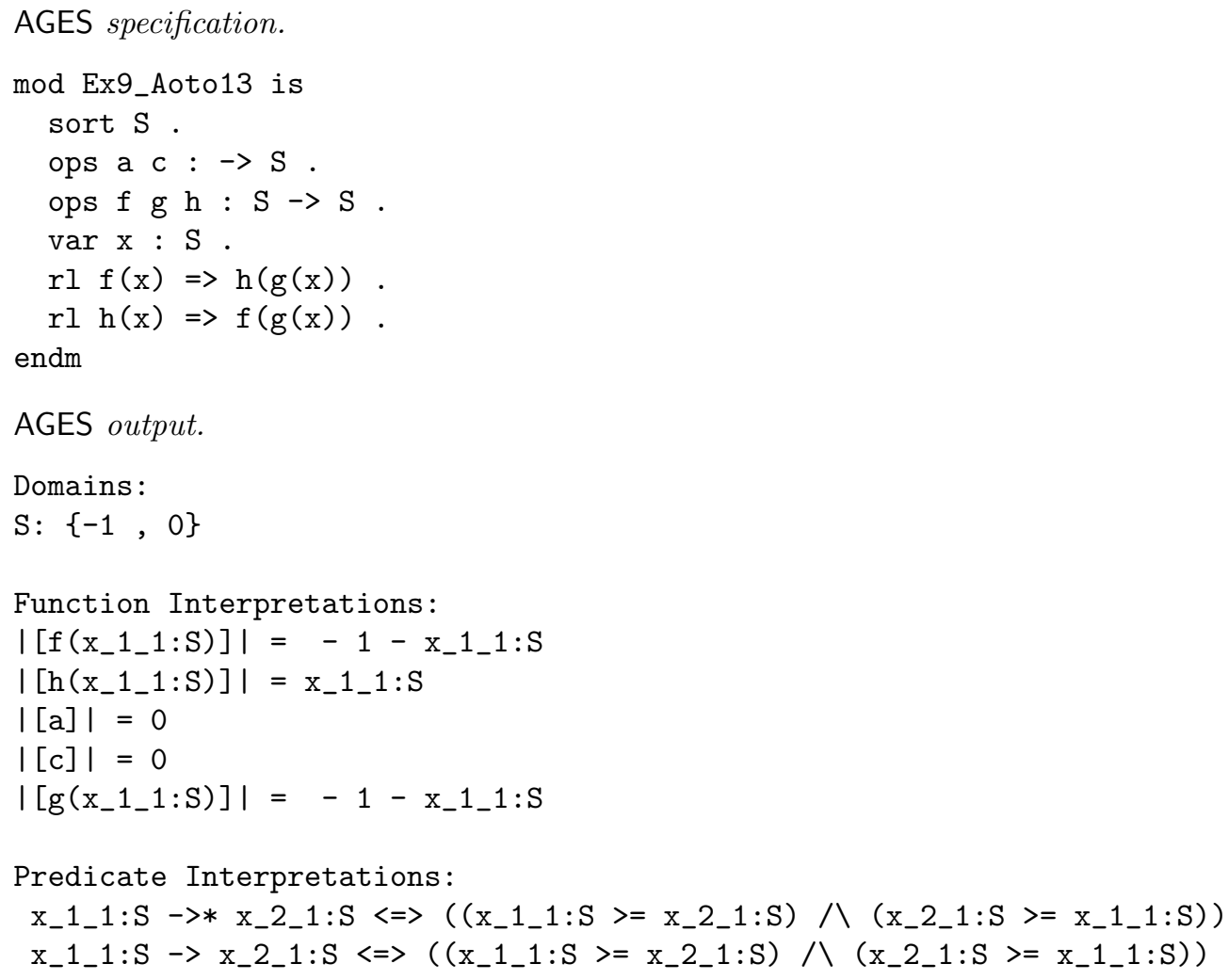

Computed model. The computed structure $\mathcal{A}$ has domain $\{-1,0\}$. Symbols are interpreted as shown above. 
Appendix B.3. Example 15 (non-joinability)

Mace4 assumptions.

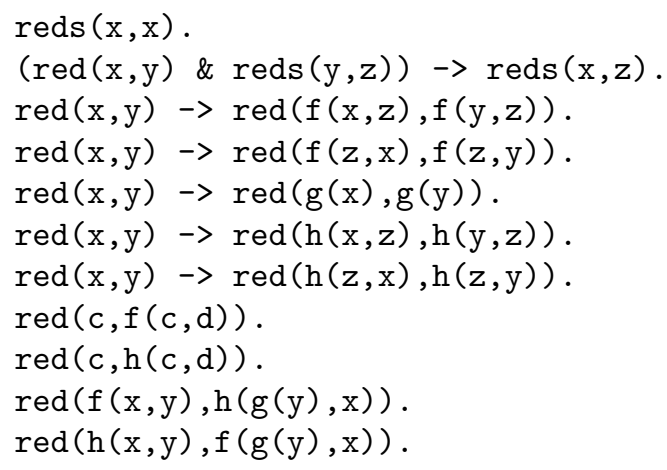

Mace4 goal.

exists $x(\operatorname{reds}(h(f(c, d), d), x) \& \operatorname{reds}(f(c, d), x))$.

Mace4 output.

$c=0$.

$\mathrm{d}=1$.

$g(0)=0$.

$g(1)=1$.

$\mathrm{g}(2)=2$.

$f(0,0)=0$.

$f(0,1)=2$.

$f(0,2)=0$.

$f(1,0)=0$.

$f(1,1)=2$.

$f(1,2)=1$.

$f(2,0)=0$.

$f(2,1)=2$.

$f(2,2)=0$.

$h(0,0)=0$.

$\mathrm{h}(0,1)=0$.

$\mathrm{h}(0,2)=0$.

$h(1,0)=2$.

$\mathrm{h}(1,1)=2$.

$\mathrm{h}(1,2)=2$.

$\mathrm{h}(2,0)=0$.

$h(2,1)=1$. 


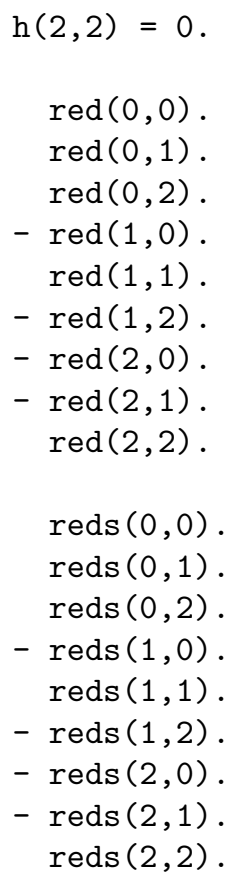

Computed model. The computed structure $\mathcal{A}$ has domain $\{0,1,2\}$. Symbols are interpreted as shown above. 


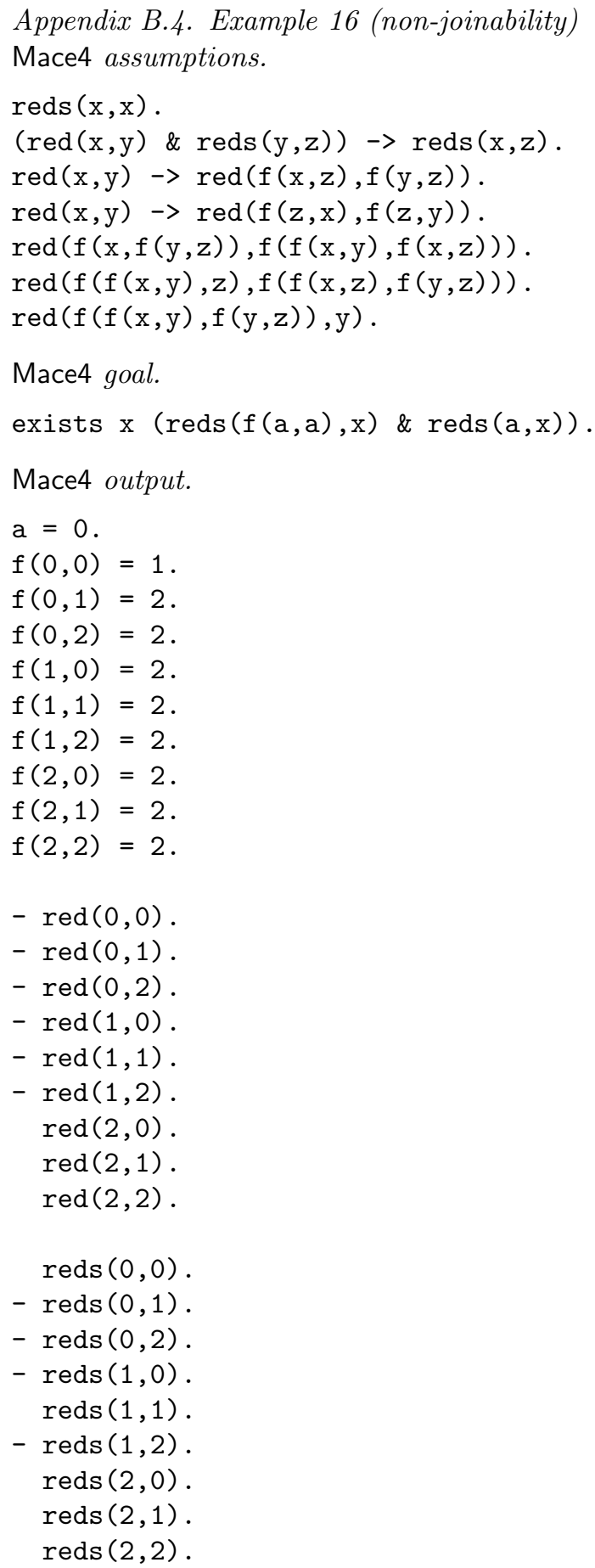

The computed structure $\mathcal{A}$ has domain $\{0,1,2\}$. Symbols interpreted as above. 


\section{Appendix C. Additional examples from [17]}

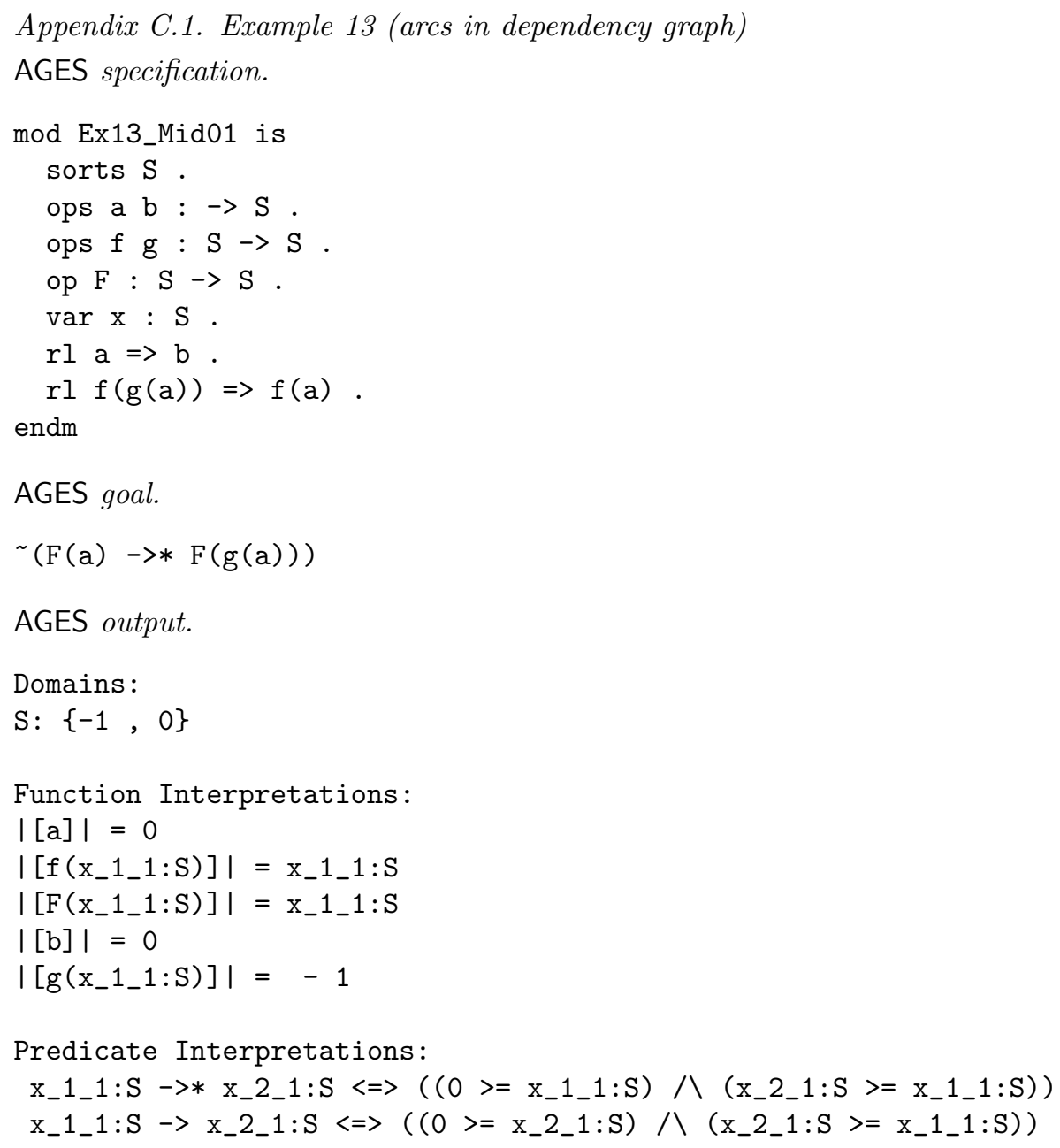

Computed model. The computed structure $\mathcal{A}$ has domain $\{-1,0\}$. Symbols are interpreted as shown above. 


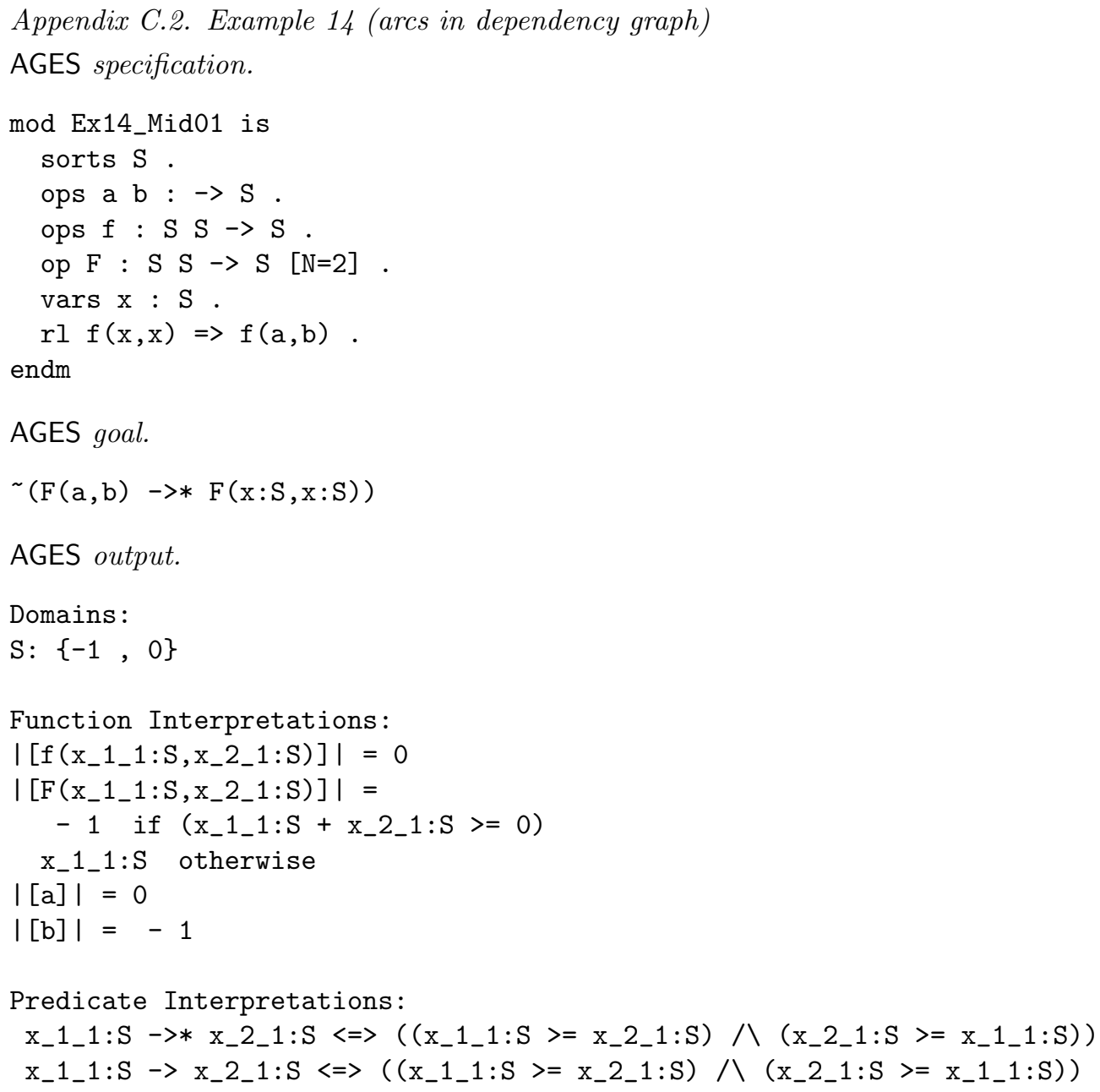

Computed model. The computed structure $\mathcal{A}$ has domain $\{-1,0\}$. Symbols are interpreted as shown above. 
Appendix C.3. Example 20 (arcs in dependency graph)

Mace4 assumptions.

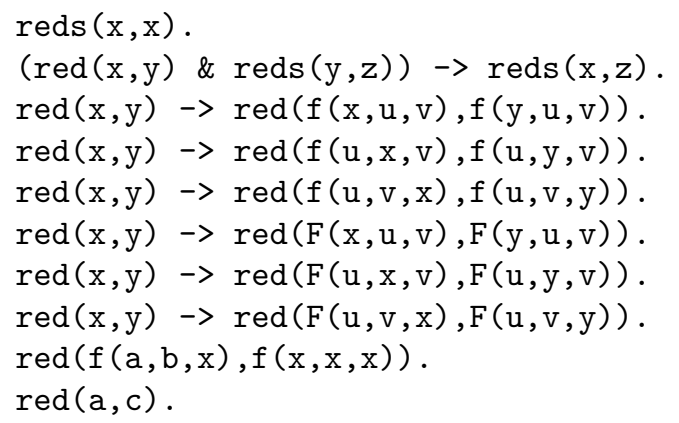

Mace4 goal.

exists $\mathrm{x}$ exists $\mathrm{y} \operatorname{reds}(\mathrm{F}(\mathrm{x}, \mathrm{x}, \mathrm{x}), \mathrm{F}(\mathrm{a}, \mathrm{b}, \mathrm{y}))$.

Mace4 output.
$\mathrm{a}=0$.
$\mathrm{b}=1$.
$c=0$.
$F(0,0,0)=0$.
$F(0,0,1)=0$.
$F(0,1,0)=1$.
$F(0,1,1)=1$.
$F(1,0,0)=0$.
$F(1,0,1)=0$.
$F(1,1,0)=0$.
$\mathrm{F}(1,1,1)=0$.
$f(0,0,0)=0$.
$f(0,0,1)=0$.
$f(0,1,0)=0$.
$f(0,1,1)=0$.
$f(1,0,0)=0$.
$f(1,0,1)=0$.
$f(1,1,0)=0$.
$f(1,1,1)=0$.
$\operatorname{red}(0,0)$.
$-\operatorname{red}(0,1)$.
$-\operatorname{red}(1,0)$. 


$$
\begin{aligned}
& \operatorname{red}(1,1) . \\
& \operatorname{reds}(0,0) . \\
- & \operatorname{reds}(0,1) . \\
- & \operatorname{reds}(1,0) . \\
& \operatorname{reds}(1,1) .
\end{aligned}
$$

Computed model. The computed structure $\mathcal{A}$ has domain $\{0,1\}$. Symbols are interpreted as shown above. 


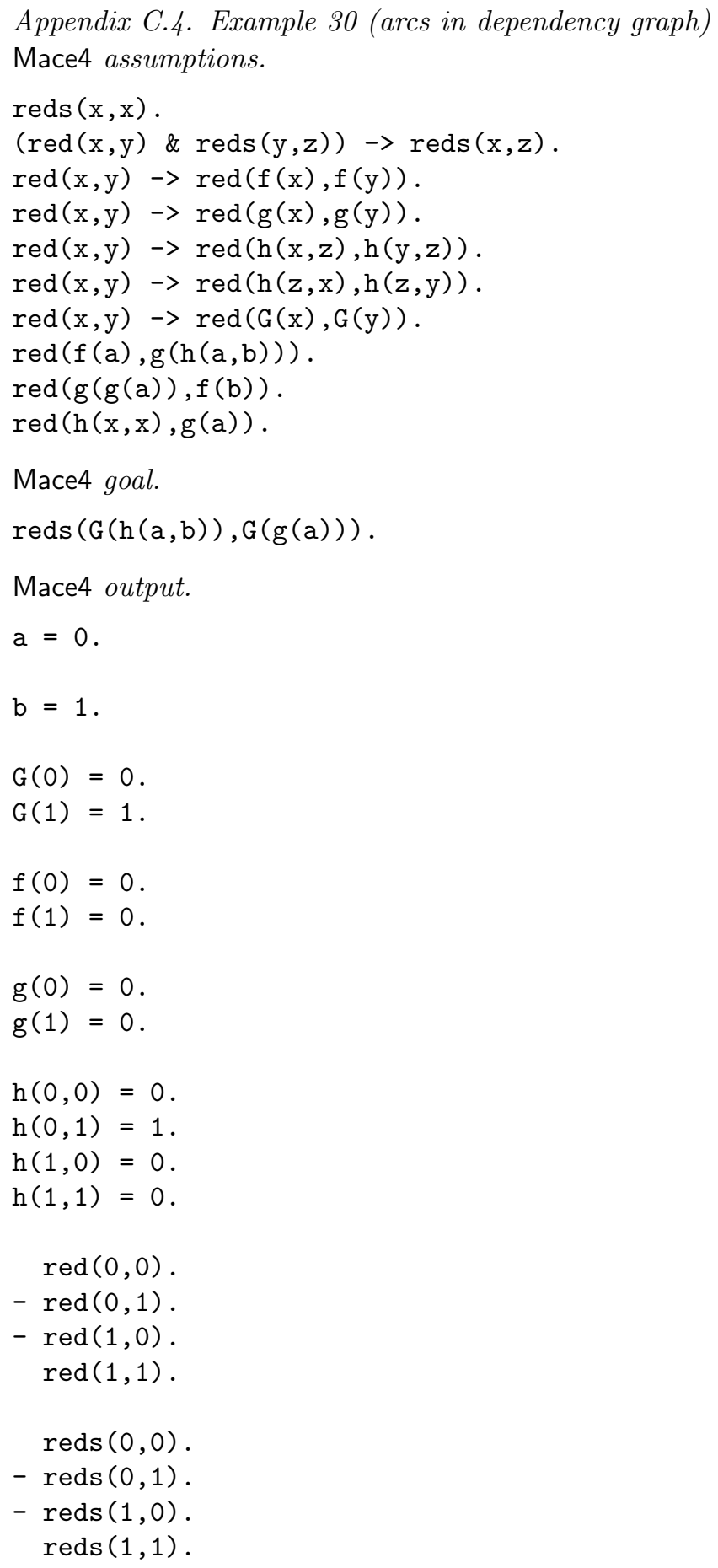

The computed structure $\mathcal{A}$ has domain $\{0,1\}$. Symbols are interpreted as above. 


\section{Appendix D. Additional examples from [20]}

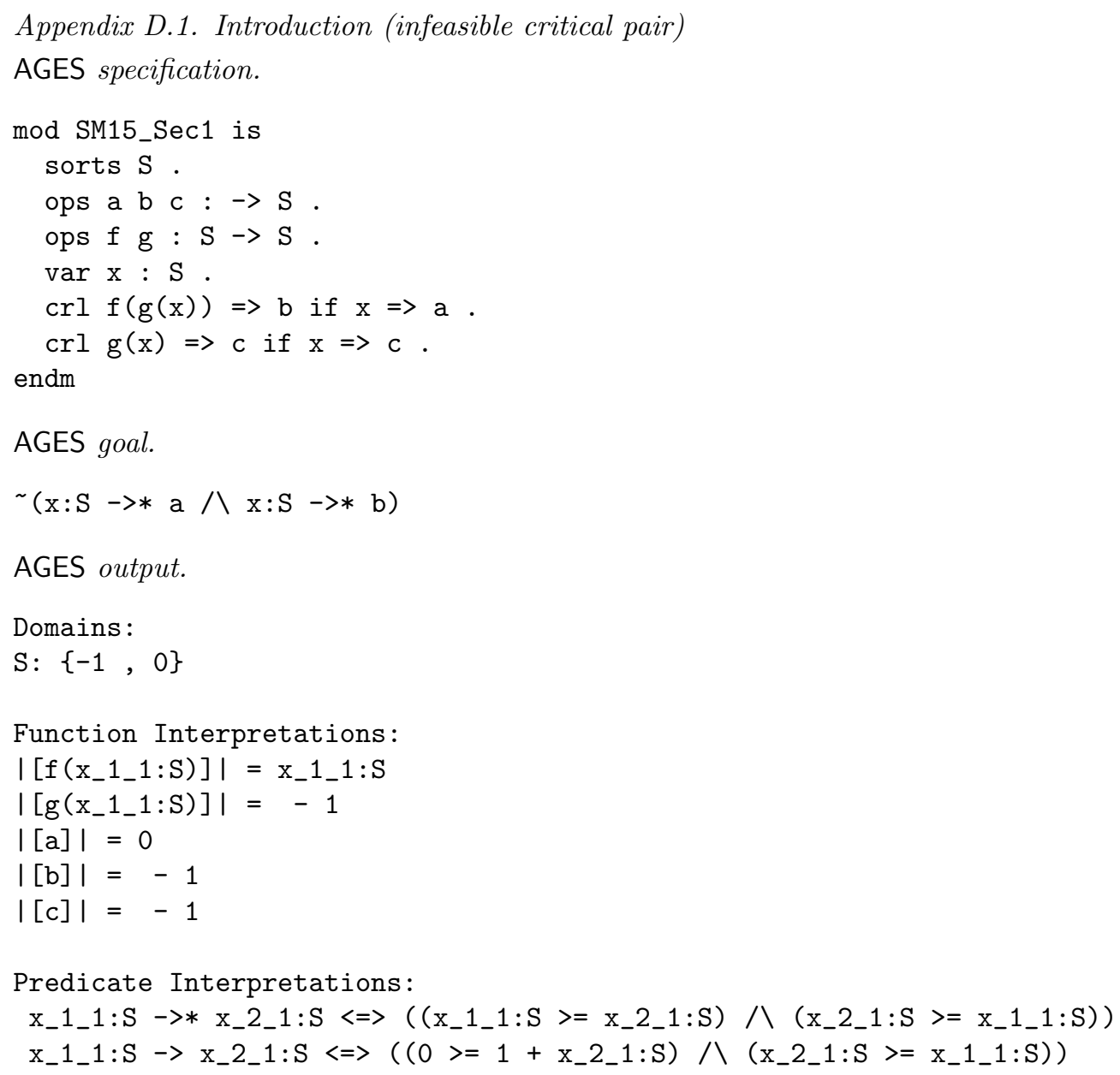

Computed model. The computed structure $\mathcal{A}$ has domain $\{-1,0\}$. Symbols are interpreted as shown above. 


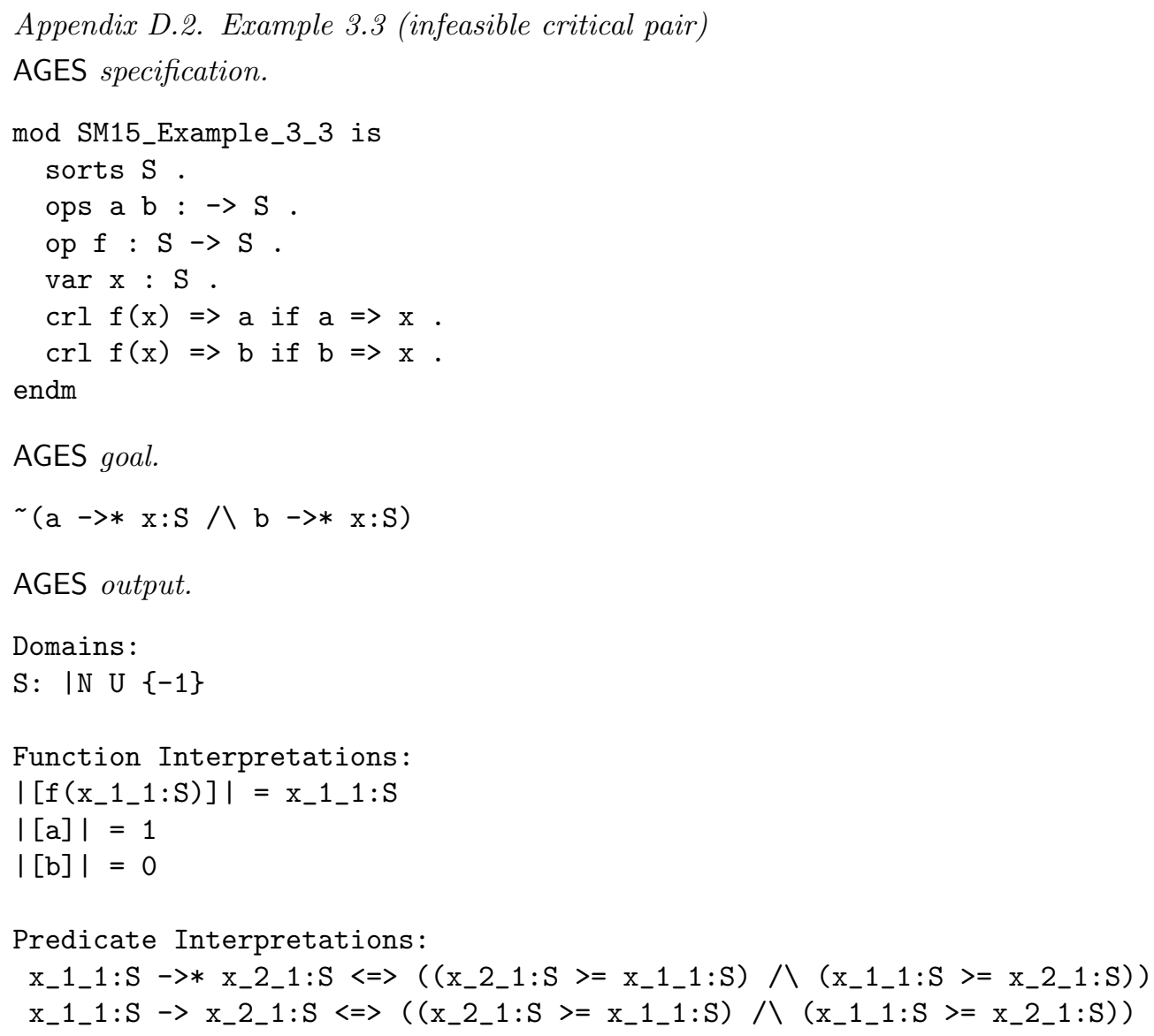

Computed model. The computed structure $\mathcal{A}$ has domain $\mathbb{N} \cup\{-1\}$. Symbols are interpreted as shown above. 
Appendix D.3. Example 5.1 (infeasible critical pairs)

AGES specification.

mod SM15_Example_5_1 is

sorts $S[m=1 \mathrm{n}=1]$.

ops Z tt : $\rightarrow S$.

op $s: S \rightarrow S$.

op leq : $\mathrm{S} S \rightarrow \mathrm{S}[\mathrm{N}=2]$.

op gt : $\mathrm{S} \mathrm{S} \rightarrow \mathrm{S}[\mathrm{N}=2]$.

op div : $S \mathrm{~S} \rightarrow \mathrm{S}$.

op minus : $\mathrm{S} S \rightarrow \mathrm{S}[\mathrm{N}=2]$.

op pair : $\mathrm{S} S \rightarrow \mathrm{S}$.

var $\mathrm{q} \times \mathrm{x}$ y : $\mathrm{S}$.

rl $\operatorname{leq}(\mathrm{Z}, \mathrm{x}) \Rightarrow \mathrm{tt}$.

rl $\operatorname{leq}(s(x), s(y)) \Rightarrow \operatorname{leq}(x, y)$.

rl $\mathrm{gt}(\mathrm{s}(\mathrm{x}), \mathrm{Z}) \Rightarrow \mathrm{tt}$.

rl $\operatorname{gt}(\mathrm{s}(\mathrm{x}), \mathrm{s}(\mathrm{y})) \Rightarrow \operatorname{gt}(\mathrm{x}, \mathrm{y})$.

$r l \operatorname{minus}(x, Z) \Rightarrow x$.

$r l$ minus $(Z, x) \Rightarrow Z$.

$r l \operatorname{minus}(s(x), s(y)) \Rightarrow \operatorname{minus}(x, y)$.

crl $\operatorname{div}(\mathrm{x}, \mathrm{y}) \Rightarrow \operatorname{pair}(\mathrm{z}, \mathrm{y})$ if $\operatorname{gt}(\mathrm{y}, \mathrm{x}) \Rightarrow \mathrm{tt}$.

crl $\operatorname{div}(x, y) \Rightarrow \operatorname{pair}(s(q), r)$ if

$\operatorname{leq}(y, x) \Rightarrow$ tt $\wedge \operatorname{div}(\operatorname{minus}(x, y), y) \Rightarrow \operatorname{pair}(\mathrm{q}, \mathrm{r})$.

endm

AGES goal.

$\sim(\operatorname{leq}(\mathrm{x}: \mathrm{S}, \mathrm{w}: \mathrm{S})->*$ tt $八 \operatorname{div}(\operatorname{minus}(\mathrm{w}: \mathrm{S}, \mathrm{x}: \mathrm{S}), \mathrm{x}: \mathrm{S}) \rightarrow * \operatorname{pair}(\mathrm{y}: \mathrm{S}, \mathrm{z}: \mathrm{S})$

$\wedge \mathrm{gt}(\mathrm{x}: \mathrm{S}, \mathrm{w}: \mathrm{S})->* \mathrm{tt})$

By Proposition 3, we can use the following simpler goal:

$\sim(\operatorname{leq}(\mathrm{x}: \mathrm{S}, \mathrm{w}: \mathrm{S})->*$ tt $八 \mathrm{gt}(\mathrm{x}: \mathrm{S}, \mathrm{w}: \mathrm{S})->* \mathrm{tt})$

AGES output. Fix the interpretation of $\rightarrow$ to be the equality.

Domains :

$\mathrm{S}: \mid \mathrm{N} \cup\{-1\}$

Function Interpretations:

$\left|\left[\operatorname{div}\left(x_{-} 1 \_1: S, x_{-} 2_{-} 1: S\right)\right]\right|=1$

$\left|\left[g t\left(x_{-} 1 \_1: S, x_{-} 2 \_1: S\right)\right]\right|=$

- 1 if ( $x_{-} 1 \_1: S>=1+x_{-} 2 \_1: S$ )

- $x_{-} 1 \_1: S+x_{-} 2 \_1: S$ otherwise

$\left|\left[\operatorname{leq}\left(\mathrm{x}_{-}{ }_{-} \_1: \mathrm{S}, \mathrm{x}_{-} 2 \_1: \mathrm{S}\right)\right]\right|=$

0 if $\left(\mathrm{x}_{-} 1 \_1: \mathrm{S}>=1+\mathrm{x}_{-} 2 \_1: \mathrm{S}\right)$

- 1 otherwise 


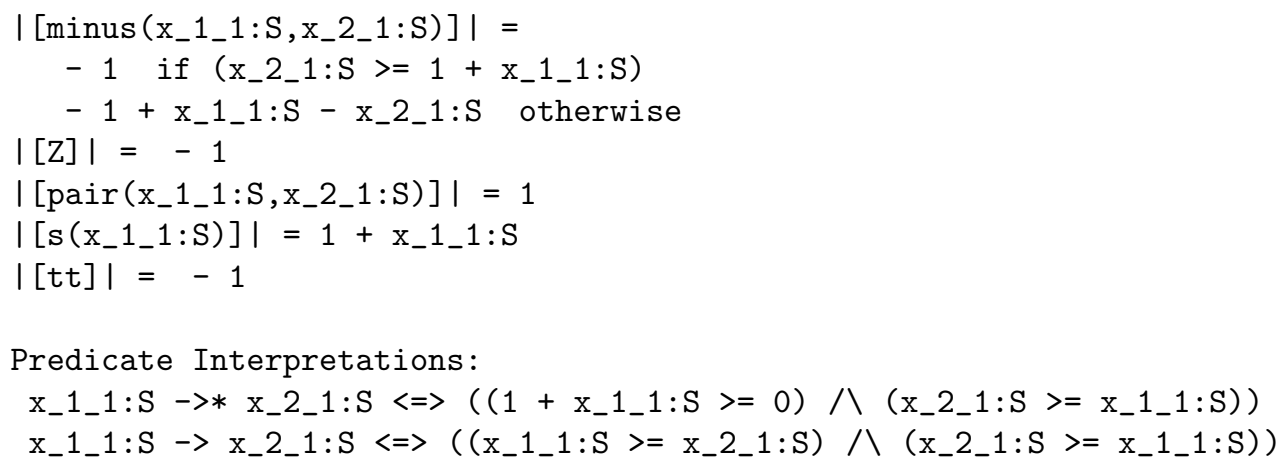

Computed model. The computed structure $\mathcal{A}$ has domain $\mathbb{N} \cup\{-1\}$. Symbols are interpreted as shown above. 


\section{Appendix E. Additional examples from [21]}

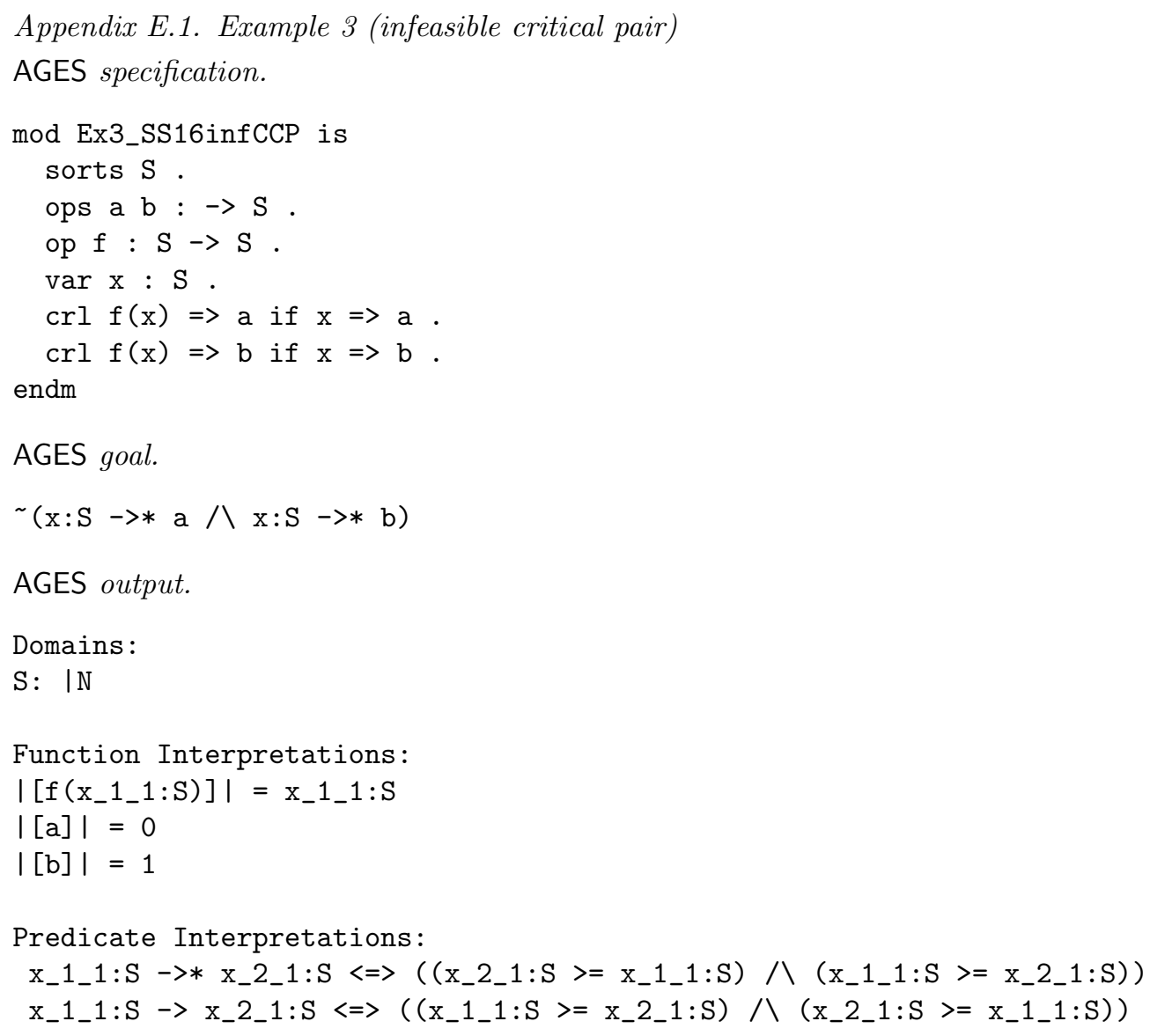

Computed model. The computed structure $\mathcal{A}$ has domain $\mathbb{N}$. Symbols are interpreted as shown above. 
Appendix E.2. Example 16 (infeasible critical pair)

AGES specification.

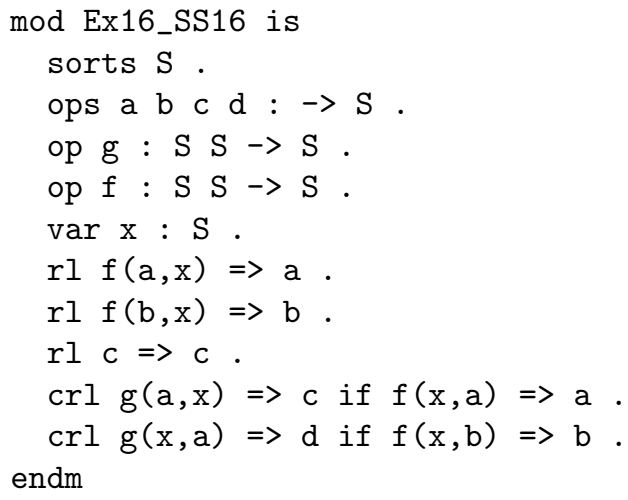

AGES goal.

$\sim(f(a, b)->* b / f(a, a)->* a)$

AGES output.

Domains :

S: |N U $\{-1\}$

Function Interpretations:

$|[c]|=-1$

$\left|\left[f\left(x_{-} 1 \_1: S, x_{-} 2 \_1: S\right)\right]\right|=x_{-} 1 \_1: S$

$\left|\left[g\left(x_{-} 1 \_1: S, x_{-} 2 \_1: S\right)\right]\right|=x_{-} 2 \_1: S$

$|[\mathrm{a}]|=-1$

$|[b]|=1$

$|[d]|=-1$

Predicate Interpretations:

$\mathrm{x}_{-} 1 \_1: \mathrm{S} \rightarrow * \mathrm{x}_{-} 2 \_1: \mathrm{S} \Leftrightarrow\left(1+\mathrm{x}_{-}{ }_{-} \_1: \mathrm{S}>=\mathrm{x}_{-} 2 \_1: \mathrm{S}\right)$

$\mathrm{x}_{-} 1 \_1: \mathrm{S} \rightarrow \mathrm{x}_{-} 2 \_1: \mathrm{S} \Leftrightarrow\left(\mathrm{x}_{-} 1 \_1: \mathrm{S}>=\mathrm{x}_{-} 2 \_1: \mathrm{S}\right)$

Computed model. The computed structure $\mathcal{A}$ has domain $\mathbb{N}$. Symbols are interpreted as shown above. 


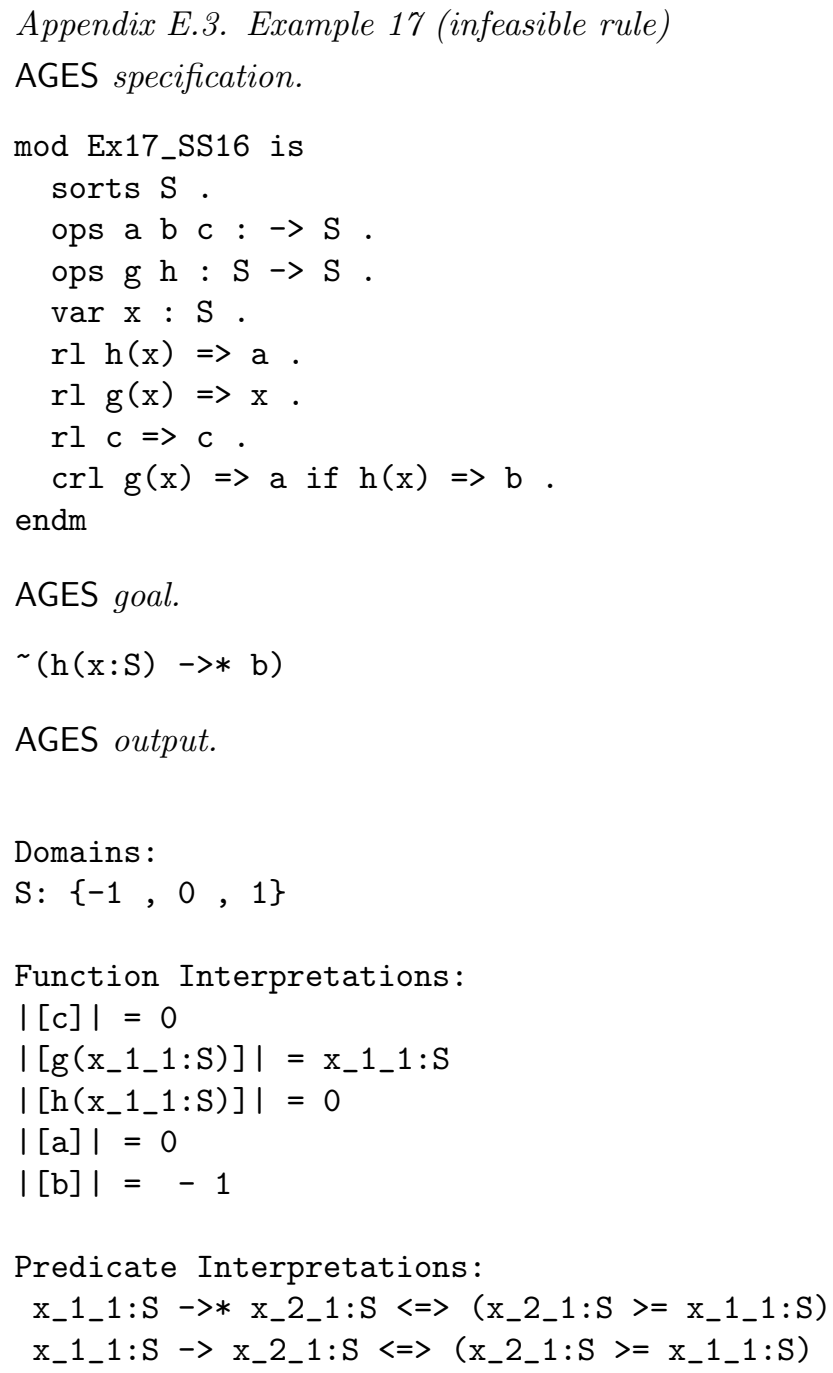

Computed model. The computed structure $\mathcal{A}$ has domain $\{-1,0,1\}$. Symbols are interpreted as shown above. 


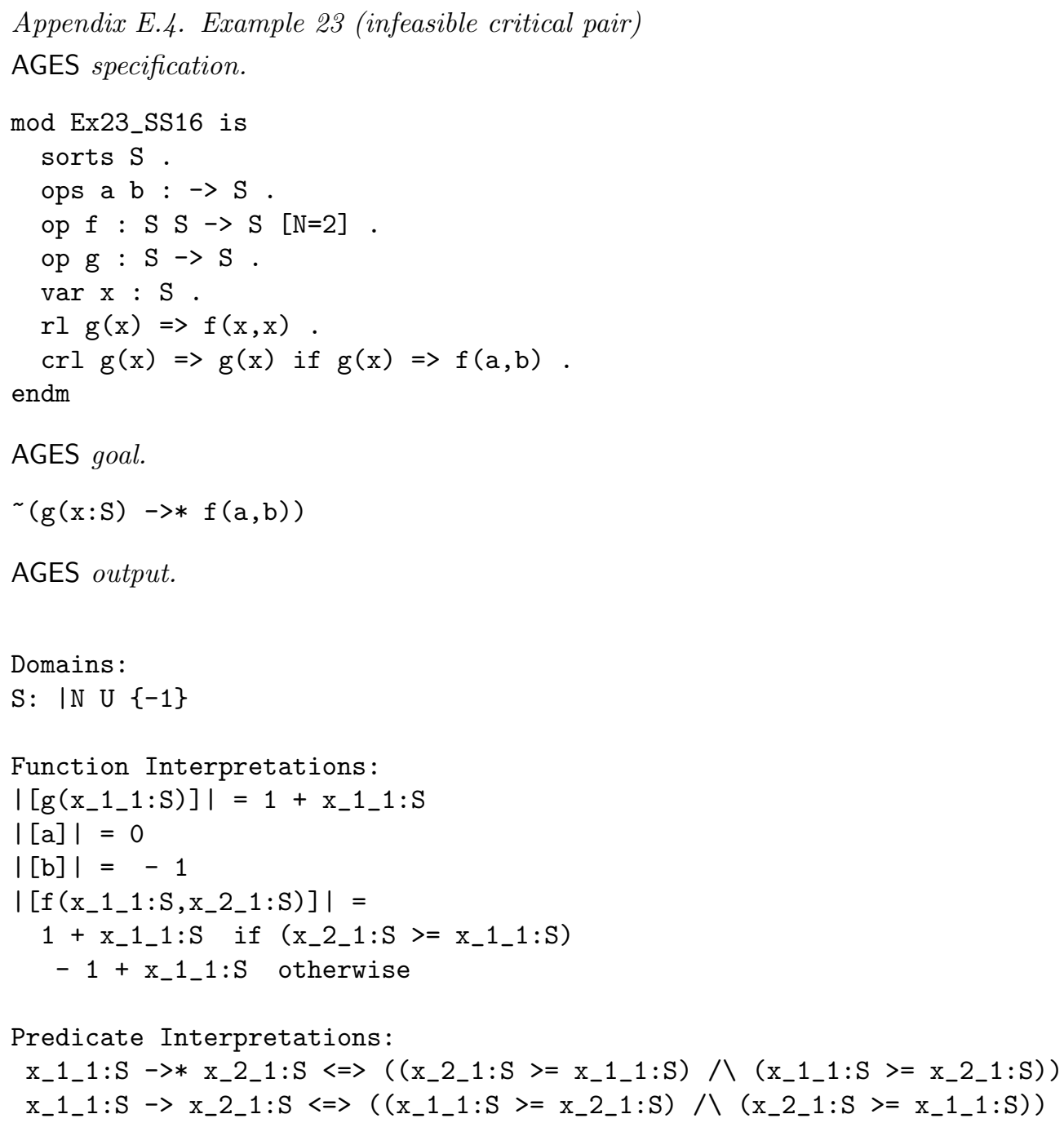

Computed model. The computed structure $\mathcal{A}$ has domain $\mathbb{N} \cup\{-1\}$. Symbols are interpreted as shown above. 